\title{
A FINITE ELEMENT APPROACH TO HÖLDER EXTENSION USING PREFRACTALS*
}

\author{
EMILY J. EVANS ${ }^{\dagger}$
}

Abstract. We extend a function $u$ which satisfies the Hölder estimate

$$
|u(x)-u(y)| \leq C_{0}|x-y|^{\beta}
$$

for all $x, y$ defined on a fractal Koch curve $S$ to a larger domain $\Omega \subset \mathbb{R}^{2}$. The extension function $u^{*}$ is defined everywhere in $\Omega$, is Hölder continuous everywhere in $\Omega$, corresponds with $u$ at every point on $S$, and satisfies the estimate $\left|u^{*}\right|_{\bar{\Omega}, \beta} \leq C\|u\|_{S, \beta}$ with a constant $C$ independent of $u$. Our approach is more constructive then the standard approach and exploits both the self-similarity of the fractal as well as the iterative process used to define the fractal set.

Key words. Koch curve, finite element, linear extension operator.

AMS subject classifications. 28A80, 65N99, 47A57.

1. Introduction. Given a function $f$ defined on a closed subset $S$ of a larger set $\Omega$ we wish to extend $f$ to the larger set such that certain characteristics of the original function $f$ are retained. The origin of the idea of analytically extending a function defined on a smaller set to a larger set goes back to the work of Whitney $[13,14,15]$ in 1934. Since the publication of Whitney's work, the problem of extending a function to a larger domain has been examined by multiple authors. Of relevance to this paper is the work of Jonsson and Wallin $[7,8,9]$ who have addressed the question of extending functions defined on special d-sets (of which the fractal Koch curve is one type) to larger domains. Their extension results rely on the use of certain trace and Besov space properties. Also relevant to this paper is the work done by Brudnyi and Shvartsman [1,2] who not only generalized Whitney's extension theorem but also investigated the existence of linear extension operators. Finally, most recently, Fefferman has also considered extension of $C^{m}$ functions where $m \geq 1$ by linear extension operators $[3,5,4,6]$.

We begin by recalling the extension results of Jonsson and Wallin [8, Theorem 3, p.155]. In this theorem $\alpha>0, B_{\beta}^{p, q}(F)$ Is the Besov space defined on the set $F$, and $\Lambda_{\alpha}^{p, q}\left(\mathbb{R}^{n}\right)$ is the Besov space on $\mathbb{R}^{n}$.

TheOREM 0. Let $F \subset \mathbb{R}^{n}$ be a d-set, $0<d \leq n, 1 \leq p, q \leq \infty, \beta=\alpha-(n-d) / p$, and $k<\beta \leq k+1$, where $k$ is a nonnegative integer. Then there is an operator $\mathcal{E}$ from $B_{\beta}^{p, q}(F)$ to $\Lambda_{\alpha}^{p, q}\left(\mathbb{R}^{n}\right)$ such that if $f=\left\{f^{(j)}\right\}_{|j| \leq k} \in B_{\beta}^{p, q}(F)$, then $\mathcal{E} f \in C^{\infty}(\bar{F})$ and

1. $\|\mathcal{E} f\|_{\Lambda_{\alpha}^{p, q}\left(\mathbb{R}^{n}\right)} \leq c\|f\|_{B_{\beta}^{p, q}(F)}$, where $c$ does not depend on $f$, and

2. $\left.D^{j}(\mathcal{E} f)\right|_{F}=f^{(j)}$ d-a.e., $|j| \leq k$.

The operator $\mathcal{E}$ is linear if $\beta$ is not an integer. It depends on $\beta$, but it is constant in the interval $k<\beta<k+1$.

The set of Hölder continuous functions defined on the fractal set $F$ is a subset of the Besov space, $B_{\beta}^{p, q}(F)$, and thus the above result gives a linear extension operator

\footnotetext{
${ }^{*}$ Received January 18, 2012; accepted for publication November 2, 2012. This research was partially supported by NSF grant DMS-1109356.

$\dagger$ Department of Mathematics, 275 TMCB, Brigham Young University, Provo, UT 84602, USA (ejevans@math.byu.edu).
} 
for Hölder continuous functions defined on a fractal set to a larger domain.

No algorithm was given by Jonsson and Wallin to prescribe the values of the extension function at points in the larger domain and we consider an explicit construction of the extension operator for Hölder continuous functions on a Koch curve. Our algorithm to prescribe values in the domain was motivated by a desire to perform numerical calculations on domains with prefractal boundaries. Recall that a prefractal curve is a polygonal curve which is a precursor to a fractal curve, assuming the fractal is constructed iteratively. A more detailed description of these curves and their construction is found in Section 2. This leads to the observation that the results presented in this paper are different from the results of Jonsson and Wallin in another key aspect; whereas Jonsson and Wallin consider extensions from only the fractal curve, we now consider the problem of determining an extension operator that is valid for both the (finite) prefractal curves and the (infinite) fractal curve.

Another important difference between our work and the work of previous authors is that we restrict to the special case of extending functions which are Hölder continuous, instead of all functions in the Besov space. This is motivated by the study of certain special boundary problems (such as the transmission problem) that have a second order condition. One unique identifying characteristic of these functions (when the boundary is a fractal boundary) is that the functions have finite energy that is intrinsic within the fractal set. These finite energy functions are Hölder continuous with a specific exponent $\beta$, which is dependent on the fractal and has value $0<\beta \leq 1$. We study the special set of nested prefractal Koch curves because they support equations which share features with the classic Laplace equations. These special sets are also relevant in connection with boundary value problems where we wish to consider 2-dimensional domains with boundaries that have dimension greater than 1. (See for example the works by Lancia, Vivaldi and Mosco [10, 11] in which certain boundary problems of transmission type are considered.)

Our approach is more constructive than previous works. In fact, our work seeks to exploit both the self-similarity of the fractal and the iterative process used to define the fractal set. In fact, we use the finite element spaces utilized when numerically approximating boundary value problems as the scaffolding of our extension. The use of finite element spaces as scaffolding is particularly helpful when numerically approximating prefractal boundary value problems because no re-approximation must occur. (In the case of previous works the extension was done on the fractal curve, but any numerical work relied on re-approximated values on the prefractal.) We construct an extension operator $\Pi_{n}$ at every step of the prefractal iteration by considering both the original function $u$ defined at the vertices of the prefractal curve and the extension operator at the previous iteration $\Pi_{n-1}$. We then construct the fractal extension operator $\Pi u^{*}$ as the limit of the sequence of prefractal extensions $u_{n}^{*}$. We say that the extension presented in this work is an adaptive extension because it easily adapts to the family of prefractals for a given fractal.

We begin with a function $u$ defined on a Koch curve $S$ which satisfies the Hölder estimate $|u(x)-u(y)| \leq C_{0}|x-y|^{\beta}$, for all $x, y$ on $S$. The constant $C_{0}$ is fixed and dependent on $u$, the minimal value of which is equal to the Hölder seminorm $|u|_{S, \beta}$. (Here $\beta$ is constant and $0<\beta \leq 1$.) What we wish to achieve is a linear extension of 
$u$ to a domain $\Omega$ with the construction of a function $u^{*}$ that is defined everywhere in $\Omega$, is Hölder continuous everywhere in $\Omega$, and corresponds with $u$ at every point on $S$. Moreover, we wish for the function to satisfy the estimate $\left|u^{*}\right|_{\bar{\Omega}, \beta} \leq C\|u\|_{S, \beta}$ with a constant $C$ independent of $u$. Here $\|u\|_{S, \beta}$ is the Hölder norm of $u$ and is defined as $\|u\|_{S, \beta}=|u|_{S, \beta}+\sup _{x \in S}|u(x)|$.

In the following theorem $\Omega$ is an arbitrary open domain in $\mathbb{R}^{2}$ such that the Koch curve $S$ is contained in $\Omega$. The main theorem of our work is:

THEOREM 1. Given a Koch curve $S$ and a domain $\Omega$ there is a linear continuous operator $\Pi$ such that for all $u \in C^{\beta}(S)$ the following holds:

1. $\Pi: C^{\beta}(S) \mapsto C^{\beta}(\Omega)$

2. $\|\Pi u\|_{\bar{\Omega}, \beta} \leq C_{1}\|u\|_{S, \beta}$

3. $\Pi u=\lim _{n \rightarrow \infty} \Pi_{n}\left(\left.u\right|_{V^{n}}\right)$ uniformly in $\Omega$

4. $\sup _{X \in \Omega}\left|\Pi_{n} u_{n}(X)-\Pi_{n+p} u_{n+p}(X)\right| \leq C_{2}\|u\|_{S, \beta} \alpha^{-n} \quad(\alpha=3)$ where $u_{n}=\left.u\right|_{V^{n}}$ and $u_{n+p}=\left.u\right|_{V^{n+p}}$.

Here $C_{1}$ and $C_{2}$ are numerical constants independent of $u$.

The format of the paper is as follows. First in Section 2, we will introduce notation related to the Koch curve and introduce an intermediate domain $\omega$. Next, we briefly describe the construction of the conformal triangulation on $\omega$ that will be the scaffolding for our extension. Then in Section 3 we will detail the methodology for extending the function $u$ to $\Omega$. In Section 4 we will present a few preliminary lemmas and their associated proofs. We then consider the iterative process used to build up the extension function in Section 5. Finally in Section 6, we prove our main theorem. In future work we will adapt the methodology developed here to different classes of fractal sets including the Sierpinski gasket, the Koch snowflake and the non-standard Koch curve.

2. The Koch curve and the triangulation of an intermediate domain. In this section we introduce some preliminary information and notation specific to the Koch curve. We also introduce an intermediate domain $\omega$ and construct a triangulation that will serve as the scaffolding for the extension.

2.1. The Koch curve. Let $\alpha \in(2,4]$ and define $\theta=\cos ^{-1}\left(\frac{\alpha}{2}-1\right)$. We consider the 4 contractive similitudes $\left\{\psi_{1}, \psi_{2}, \psi_{3}, \psi_{4}\right\}$ in $\mathbb{R}^{2}$ :

$$
\begin{array}{ll}
\psi_{1}(z)=\frac{z}{\alpha} & \psi_{2}(z)=\frac{z}{\alpha} e^{i \theta}+\frac{1}{\alpha} \\
\psi_{3}(z)=\frac{z}{\alpha} e^{-i \theta}+\frac{1}{2}+\frac{i \sin \theta}{\alpha} & \psi_{4}(z)=\frac{z+\alpha-1}{\alpha}
\end{array}
$$

where $z \in \mathbb{C}$.

For each integer $n>0$, we will consider arbitrary n-tuples of indices $i \mid n=\left(i_{1}, i_{2}, \ldots, i_{n}\right) \in\{1,2,3,4\}^{n}$. We then define $\psi_{i \mid n}=\psi_{i_{1}} \circ \psi_{i_{2}} \circ \cdots \circ \psi_{i_{n}}$ and for any set $\mathcal{G}\left(\subseteq \mathbb{R}^{2}\right), \mathcal{G}_{i \mid n}=\psi_{i \mid n}(\mathcal{G})$.

Let $V_{0}=\{A, B\}$. For every integer $n>0$, let

$$
V^{n}=\bigcup_{i \mid n} V_{0}^{i \mid n}
$$


where $V_{0}^{i \mid n}=\left(V_{0}\right)^{i \mid n}$ in the preceding notation. We put

$$
V^{\infty}=\bigcup_{n=0}^{+\infty} V^{n}
$$

and

$$
S=\bar{V}^{\infty}
$$

the closure being in $\mathbb{R}^{2}$. The set $S$ is the Koch curve in $\mathbb{R}^{2}$ and has Hausdorff dimension of $(\ln 4) /(\ln \alpha)$. We note that $V^{n} \subset V^{\infty} \subset S$ and that as $n$ increases the number of points in $V^{n}$ also increases. We will denote by $S^{n}$ the prefractal (polygonal) curve of iteration number $n$ created by connecting the points in the set $V^{n}$ in a prescribed manner. Although the Koch curve exists for all values of $\alpha$ such that $2<\alpha \leq 4$, we restrict ourselves to only the standard case where $\alpha=3$.

REMARK 1. We note that the prefractal polygonal curves of different iteration number are not nested. That is to say $S^{n-1} \subsetneq S^{n} \subsetneq S$. For this reason we work with the set of vertices $V^{n}$ correspondent to each curve $S^{n}$.

2.2. Induced triangulation of an intermediate domain. We define $\omega$ as the polygonal domain with vertices $A=(0,0), B=(1,0), C=\left(\frac{1}{2}, \frac{\sqrt{3}}{2}\right)$, and $D=\left(\frac{1}{2}, \frac{-\sqrt{3}}{2}\right)$, and $S$ the fractal Koch curve with end points $A$ and $B$. Additionally we will require that the vertices of the prefractal set $V^{n}$ are nodes of the triangulation $T_{V^{n}}$ (that is to say the triangulation induced by the prefractals set $V^{n}$ ). We will use the same triangulation defined previously in works by Lancia and Vivaldi [10] and Vacca [12]. We define the initial triangulation $T_{V^{1}}$ as follows:

We start by constructing the equilateral triangle with vertices $(1 / 3,0),(2 / 3,0)$, $(1 / 2, \sqrt{3} / 6)$ and we proceed constructing five other equal triangles such that the union of the six equilateral triangles gives the regular hexagon centered in $(1 / 2, \sqrt{3} / 6)$. The triangle $A B C$ is the union of 9 congruent equilateral triangles. By proceeding in a symmetric way in the triangle $A B D$ we complete the triangulation of $\omega$.

By proceeding in this regular way we can construct the triangulation $T_{V^{n+1}}$ of $\omega$ by subdividing each triangle of $T_{V^{n}}$ into nine congruent subtriangles.

3. A methodology for the extension. We construct the extension function on the domain $\Omega$ in an iterative manner. We begin by constructing a base extension function $u_{0}^{*}=\Pi_{0} u_{0}$ from the function $u_{0}$; we then construct the function $u_{1}^{*}=\Pi_{1} u_{1}$, using information from both $u_{1}$ and $u_{0}^{*}$. We continue in a step by step manner constructing the extension function $u_{n}^{*}$ from $u_{n}$ and $u_{n-1}^{*}$.

Before continuing with our extension methodology we wish to define what we mean when we refer to the sidecar triangles of the prefractal set $V^{n}$ and to the transition triangles of the prefractal set $V^{n}$.

Definition 1. Given a prefractal set $V^{n}$ we define the triangles which share one or more vertices with the vertices of the polygonal prefractal curve to be the sidecar triangles. See the light gray triangles in Figure 1. 
Definition 2. Given a prefractal set $V^{n}$ we define the triangles which share one or more vertices with the vertices of the sidecar triangles of $V^{n}$ to be the transition triangles. See the white triangles in Figure 1.

3.0.1. Extending to $\omega$. For a given set $V^{n}$ we divide $\omega$ into three subdomains: $T_{S C}^{n}, T_{T R}^{n}$ and $T_{E X}^{n}$. The subdomain $T_{S C}^{n}$ is composed of the set of sidecar triangles for the set $V^{n}$. The subdomain $T_{T R}^{n}$ is composed of the set of transition triangles of $V^{n}$. Finally, the subdomain $T_{E X}^{n}$ is the set of all the triangles in the domain $\omega$ which are neither sidecar triangles nor transition triangles (i.e $T_{E X}^{n}=\omega \backslash\left(T_{S C}^{n} \cup T_{T R}^{n}\right)$ ). This division is illustrated in Figure 1 where the light gray triangles are $T_{S C}^{n}$, the white triangles are $T_{T R}^{n}$, and the dark triangles are $T_{E X}^{n}$. We will consider these three domains separately when constructing $u_{n}^{*}$.

The Base Extension. We begin by constructing $u_{0}^{*}$. For this case we do not subdivide $\omega$ into three domains, but rather treat $\omega$ as the union of two sidecar triangles. To construct $u_{0}^{*}$ we set $u_{0}^{*}(C)=u_{0}^{*}(D)=\frac{1}{2}(u(A)+u(B))$. We then set $u_{0}^{*}$ to be the affine function determined by the values of $u_{0}^{*}$ at the three vertices of the triangles $A B C$ and $A B D$ respectively; i.e., $u_{0}^{*}(x, y)=\frac{u(B)-u(A)}{|B-A|} x+u(A)$. (See Figure 1).

To construct the extension function $u_{n}^{*}$ in $\omega$ we use values of $u$ at the vertices of the set $V^{n}$ and values of $u_{n-1}^{*}$ from the domain $\omega$. First we obtain the triangulation of $\omega$ induced by $V^{n}$. We then consider the three subdomains defined earlier and construct the extension as follows:

Extending to $T_{S C}^{n}$. First we identify the prefractal set $V^{n}$ and the sidecar triangles associated with this set. We observe that each triangle in $T_{S C}^{n}$ has one or more vertices that are elements of $V^{n}$ and hence have values of $u$ defined at these points. Therefore the value of $u_{n}^{*}$ is predetermined at one, two or three vertices of each triangle. We will divide these triangles into three distinct groups. The first group (primary sidecar triangles) will contain those sidecar triangles which have two or three vertices which are elements of $V^{n}$. The second group (secondary sidecar triangles) will contain those sidecar triangles which have one vertex which is an element of $V^{n}$ and one or two vertices which are also a vertex of a primary sidecar. The third group (tertiary sidecar triangles) will contain those sidecar triangles which have a single vertex in $V^{n}$ and no vertices shared by a primary sidecar.

We first extend $u$ to $u_{n}^{*}$ in the primary sidecar triangles. We do this by identifying the vertices in the primary sidecar triangles where $u_{n}^{*}$ is undetermined. Let $J$ be one of the vertices where $u_{n}^{*}$ is undetermined, and $\mathcal{T}_{J}$ be the set of primary sidecar triangles which have $J$ as a vertex. We set $u_{n}^{*}$ at $J$ to be the average of the values of $u$ at each element of $V^{n}$ which is also a vertex of one of the triangles of $\mathcal{T}_{J}$. Now each primary sidecar triangle has $u_{n}^{*}$ determined at every vertex and we set the extension function $u_{n}^{*}$ to be the affine function on the triangle determined by the values of $u_{n}^{*}$ at the three vertices.

We now extend $u_{n}^{*}$ to the secondary sidecar triangles. We proceed in a manner similar to that of the primary sidecar triangles by first identifying the vertices in the secondary sidecar triangles where $u_{n}^{*}$ is undetermined. Again, we let $J$ be one of the 
vertices where $u_{n}^{*}$ is undetermined, and $\mathcal{T}_{J}$ be the set of secondary sidecar triangles which have $J$ as a vertex. We set $u_{n}^{*}$ at $J$ to be the average of the values of $u_{n}^{*}$ at each vertex of the triangles $\mathcal{T}_{J}$ where the value of $u_{n}^{*}$ is predetermined. We then set the function $u_{n}^{*}$ to be the affine function on the triangle determined by the value of $u_{n}^{*}$ at the vertices. Having performed this extension on the secondary sidecar triangles, $u_{n}^{*}$ will be predetermined at each vertex of the tertiary sidecar triangles. We set the extension inside these triangles to be the affine function determined by the values of $u_{n}^{*}$ at the vertices of the triangle.

REMARK 2. We note that the maximum depth of averaging is two. That is to say, in order to find the value of $u_{n}^{*}$ at the vertex of a sidecar triangle we may have to perform two averaging steps (one for those in the primary sidecars, and one for the secondary sidecars) but we never perform three averaging steps. This maximum depth is independent of the iteration number $n$.

Extending to $T_{T R}^{n}$. Prior to this step $u$ has been extended to $u_{n}^{*}$ in $T_{S C}^{n}$, and hence values for $u_{n}^{*}$ are predetermined at every vertex common between $T_{S C}^{n}$ and $T_{T R}^{n}$. Additionally, the function $u_{n-1}^{*}$ is defined everywhere on the domain $\omega$. Extension to $T_{T R}^{n}$ is done on a triangle by triangle basis by setting the value of $u_{n}^{*}$ to be equal to the value of $u_{n-1}^{*}$ at any vertex where the value of $u_{n}^{*}$ is not predetermined. We then set $u_{n}^{*}$ to be the function which is affine on the triangle and determined by the values of $u_{n}^{*}$ at the three vertices of the triangle.

Extending to $T_{E X}^{n}$. The extension to $T_{E X}^{n}$ is accomplished by setting $u_{n}^{*}$ equal to $u_{n-1}^{*}$ at every point in $T_{E X}^{n}$.

In this manner we proceed in an iterative fashion to construct each function $u_{n}^{*}$ on $T_{V^{n}}$. We summarize the extension methodology with the following definition

Definition 3. The extension function $u_{n}^{*}$ on $\omega$ is defined as the function determined by the values of $u$ on $V^{n}$ and the values of the previous function $u_{n-1}^{*}$ on $\omega$. The values of $u$ on $V^{n}$ are used to determine the function $u_{n}^{*}$ on the sidecar triangles of $\omega$, the values of $u$ on $V^{n}$ and the values of $u_{n-1}^{*}$ are used to determine the function $u_{n}^{*}$ on the transition region of $\omega$, and finally only the values of $u_{n-1}^{*}$ are used to determine the function $u_{n}^{*}$ on the region of $\omega$ which is neither sidecar or transition region.

This definition is shown visually for steps $0,1,2$, and 3 in Figure 1.

3.0.2. Extending to $\Omega$. We introduce an additional intermediate domain $\gamma$ where $\gamma$ is the convex polygonal domain with vertices $E=(-1,0), \quad F=$ $(0, \sqrt{3}), \quad G=(1, \sqrt{3}), \quad H=(2,0), \quad I=(1,-\sqrt{3})$, and $J=(0,-\sqrt{3})$. We subdivide $\gamma$ into sixteen congruent triangles, two of which are the triangles $A B C$ and $A B D$, which composed the domain $\omega$. The function $v_{n}^{*}$ is constructed on $\gamma$ by a combination of operations of rotation, reflection and translation applied to the function $u_{n}^{*}$ defined on the two triangles $A B C$ and $A B D$. These operations are shown visually in Figure 2. In this figure, the light gray triangles indicate those triangles where the value of $v_{n}^{*}$ is obtained by translation and rotation of the function $u_{n}^{*}$ defined on $A B C$ and the lined triangles are obtained by translation and rotation of the function $u_{n}^{*}$ defined on the triangle $A B D$. The dark gray triangles are obtained by first reflecting the triangle $A B C$ across the $x$-axis and then translating and rotating the reflected function. Similarly the dotted triangles 


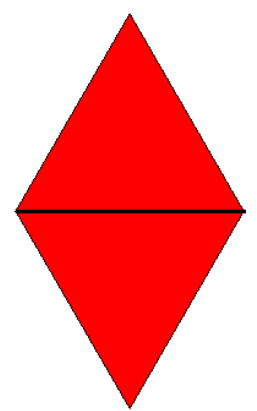

(a)

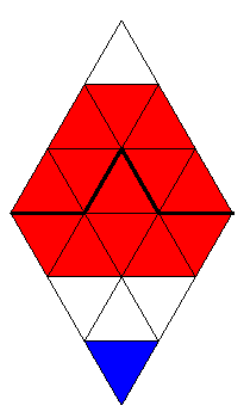

(b)

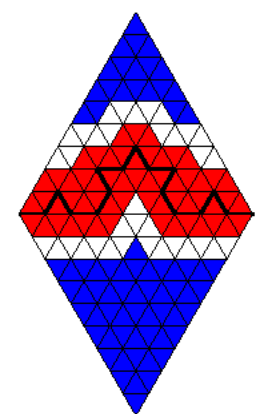

(c)

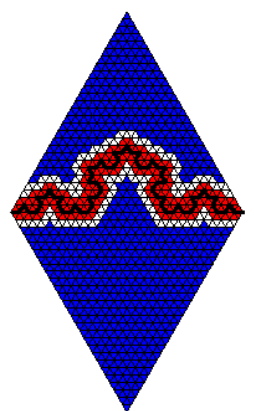

(d)

FIG. 1. Steps 0 -3 of the extension methodology. The light gray triangles are the sidecar triangles $\left(T_{S C}^{n}\right)$, the white triangles are the transition triangles $\left(T_{T R}^{n}\right)$ and the dark triangles are external triangles $\left(T_{E X}^{n}\right)$.

are obtained by reflecting the function $u_{n}^{*}$ in $A B D$ across the $x$-axis then translating and rotating the function. Clearly if the function $u_{n}^{*} \in C^{\beta}(\omega)$, then $v_{n}^{*} \in C^{\beta}(\gamma)$.

We will utilize the standard finite element "hat" function in relationship to the sixteen triangle coarse mesh. The function $\eta_{A}$ corresponds to the function with value 1 at point $A$, zero on the boundary of the hexagon created by the six triangles with $A$ as a vertex, and affine on each of the triangles. In a similar manner we create functions $\eta_{B}, \eta_{C}$, and $\eta_{D}$ centered at points $B, C$, and $D$ respectively. We set $\eta=$ $\eta_{A}+\eta_{B}+\eta_{C}+\eta_{D}$, and observe that $\eta \in H^{1}(\gamma), \eta(X)=1$ for all $X \in \omega$, and $\eta(X)=0$ for all $x \in \partial \gamma$. We define the function $z_{n}^{*}(X)=\eta(X) v_{n}^{*}(X)$ for $X \in \gamma$ and $z_{n}^{*}(X)=0$ for $X \in \mathbb{R} \backslash \gamma$. We set the extension function $u_{n}^{*}$ to $z_{n}^{*}$ and restrict $u_{n}^{*}$ to the given domain $\Omega$.

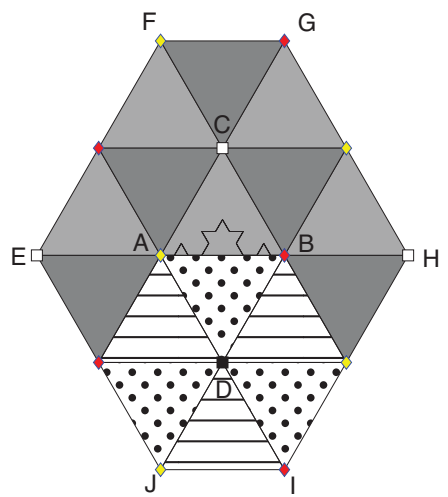

FIG. 2. An illustration of $\gamma$ and the sixteen subtriangles. The patterned triangles are reflections and translations of $A B D$ and the gray triangles are reflections and translations of $A B C$. The white diamonds indicate those points where $v_{n}^{*}$ is equal to $u(A)$, the gray diamonds those points where $v_{n}^{*}$ is equal to $u(B)$, the white squares $v_{n}^{*}=u_{n}^{*}(C)$ and the black square where $v_{n}^{*}=u_{n}^{*}(D)$.

Proposition 1. The extension operator $\Pi_{n}$ is linear. 
Proof. Let $S$ be a Koch curve and $u, v$ be two Hölder continuous functions defined at the vertices on the curve that satisfy $|u(X)-u(Y)| \leq|u|_{S, \beta}|X-Y|^{\beta}$, and $|v(X)-v(Y)| \leq|v|_{S, \beta}|X-Y|^{\beta}$, for all $X, Y \in V^{n}$ and for some $\beta \leq 1$. We also let $\kappa, h \in \mathbb{R}$. First, we assume $X$ is an element of $\omega$. By construction the value of $\Pi_{n} u_{n}(X)$ is the linear combination of the values of $\Pi_{n} u_{n}\left(X_{1}\right), \Pi_{n} u_{n}\left(X_{2}\right)$, and $\Pi_{n} u_{n}\left(X_{3}\right)$, where $X_{1}, X_{2}$, and $X_{3}$ are the vertices of the triangle $\mathcal{T}_{X}$. Also by construction the value of $\Pi_{n} u_{n}\left(X_{i}\right)=\sum_{i=1}^{\left|V^{n}\right|} a_{i} u\left(y_{i}\right)$ where $y_{i} \in V^{n}, 0 \leq a_{i} \leq 1, \sum a_{i}=1$ and the values of $a_{i}$ are independent of $u$. Therefore:

$$
\begin{aligned}
\kappa \Pi_{n} u_{n}(X)+h \Pi_{n} v_{n}(X) & =\kappa \sum_{i=1}^{\left|V^{n}\right|} a_{i} u\left(y_{i}\right)+h \sum_{i=1}^{\left|V^{n}\right|} a_{i} v\left(y_{i}\right) \\
& =\sum_{i=1}^{\left|V^{n}\right|} a_{i}\left(\kappa u\left(y_{i}\right)+h v\left(y_{i}\right)\right) \\
& =\prod_{n}\left(\kappa u_{n}(X)+h v_{n}(X)\right) .
\end{aligned}
$$

4. Preliminary results. The purpose of this section is to provide a few preliminary results needed for the proof of Theorem 1 for the Koch curve. The first lemma is specific to the primary sidecar triangles, the second to the secondary sidecar triangles and the third specific to the tertiary sidecar triangles. Lemmas 4-5 consider the Hölder estimate on two or more triangles.

LEMma 1. Given a triangle $\mathcal{T}$ with vertices $P, Q$, and $J$, and a function $u$ defined at the vertices of the prefractal $V^{n}$ such that $|u(A)-u(B)| \leq|u|_{S, \beta}|A-B|^{\beta}$ where $A, B \in V^{n}$ and $\beta \leq 1$. Moreover $P, Q \in V^{n}$. Then there exists an extension function $u^{*}$ defined on $\mathcal{T}$ such that

$$
\sup _{X, Y \in \mathcal{T}} \frac{\left|u^{*}(X)-u^{*}(Y)\right|}{|X-Y|^{\beta}} \leq 3|u|_{S, \beta} .
$$

Proof. We consider the case where the value of $u^{*}$ at point $J$ is determined by the value of $u$ at five vertices of the prefractal curve. (The case where $u^{*}$ at point $J$ is determined by two, three or four vertices proceeds in a similar manner, with the right hand side of (1) equal to $a|u|_{S, \beta}$, with $a \rightarrow 1$ as the number of vertices decreases). We let $P, Q, R, S$ and $T$ be five consecutive vertices along the prefractal curve. (See Figure 3 for an illustration of the scenario considered.) We begin by constructing the extension function $u^{*}$, by prescribing its value at $J$. We set $u^{*}(J)=\frac{1}{5}(u(P)+u(Q)+u(R)+u(S)+u(T))$ and let $u^{*}$ be the affine function defined by these three points. We now check that $u^{*}$ satisfies the estimate $(1)$.

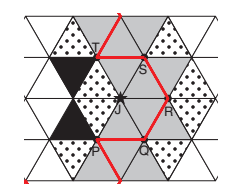

FIG. 3. The triangle $P Q J$ under consideration 
To facilitate our calculations we introduce a reference triangle $\hat{\mathcal{T}}$. The triangle $\hat{\mathcal{T}}$ is the equilateral triangle with $\hat{P}$ located at the origin, $\hat{Q}$ located on the $x$-axis, and $|\hat{P}-\hat{Q}|=|P-Q|$. With this triangle we associate the map $\tau: \hat{X} \rightarrow X$ in $\mathbb{R}^{2}$ which brings the vertex $\hat{P}$ to $P$, and the vertex $\hat{Q}$ to $Q$. Here $\tau \hat{X}=x_{0}+B \hat{X}$ where $x_{0}$ is a $2 \times 1$ vector and $B$ is the rotational matrix with $\theta=-\arctan \left[\left(y_{Q}-y_{P}\right) /\left(x_{Q}-x_{P}\right)\right]$. We note that $|X-Y|=|\hat{X}-\hat{Y}|$ and observe that

$$
\sup _{X, Y \in \mathcal{T}} \frac{\left|u^{*}(X)-u^{*}(Y)\right|}{|X-Y|^{\beta}}=\sup _{\hat{X}, \hat{Y} \in \hat{\mathcal{T}}} \frac{\left|\hat{u}^{*}(\hat{X})-\hat{u}^{*}(\hat{Y})\right|}{|X-Y|^{\beta}} .
$$

Therefore it suffices to show the estimate in $\hat{\mathcal{T}}$. It can easily be checked that the function $u^{*}$ satisfies

$$
\hat{u}^{*}(\hat{X})=\frac{u(Q)-u(P)}{|P-Q|} \hat{x}+\frac{2 u(J)-u(P)-u(Q)}{\sqrt{3}|P-Q|} \hat{y}+u(P) .
$$

We also observe that

$$
\hat{u}^{*}(\hat{J})=\frac{1}{5}(u(P)+u(Q)+u(R)+u(S)+u(T)) .
$$

Let $\hat{X}_{1}=\left(\hat{x}_{1}, \hat{y}_{1}\right)$ and $\hat{X}_{2}=\left(\hat{x}_{2}, \hat{y}_{2}\right)$ be arbitrary points in $\hat{\mathcal{T}}$.

$$
\begin{aligned}
& \frac{\left|\hat{u}^{*}\left(\hat{X}_{1}\right)-\hat{u}^{*}\left(\hat{X}_{2}\right)\right|}{\left|\hat{X}_{1}-\hat{X}_{2}\right|^{\beta}} \\
& =\frac{\left|\frac{u(P)-u(Q)}{|P-Q|}\left(\hat{x}_{1}-\hat{x}_{2}\right)+\frac{2 u(J)-u(P)-u(Q)}{\sqrt{3}|P-Q|}\left(\hat{y}_{1}-\hat{y}_{2}\right)\right|}{\left|\hat{X}_{1}-\hat{X}_{2}\right|^{\beta}} \\
& \leq \frac{|u(P)-u(Q)|}{|P-Q|^{\beta}}+\frac{\mid 2\left(\frac{1}{5}(u(P)+u(Q)+u(R)+u(S)+u(T))-u(P)-u(Q) \mid\right.}{\sqrt{3}|P-Q|^{\beta}} \\
& \leq|u|_{S, \beta}+\frac{2}{5 \sqrt{3}}\left(\frac{|u(S)-u(P)|}{|P-Q|^{\beta}}+\frac{|u(R)-u(Q)|}{|P-Q|^{\beta}}\right)+ \\
& \quad+\frac{1}{5 \sqrt{3}}\left(\frac{|u(T)-u(P)|}{|P-Q|^{\beta}}+\frac{|u(T)-u(Q)|}{|P-Q|^{\beta}}\right) \\
& \leq|u|_{S, \beta}+\frac{2^{(1+\beta)}}{5 \sqrt{3}} \frac{|u(S)-u(P)|}{|S-P|^{\beta}}+\frac{2}{5 \sqrt{3}} \frac{|u(R)-u(Q)|}{|R-Q|^{\beta}}+ \\
& \quad+\frac{3^{\beta / 2}}{5 \sqrt{3}} \frac{|u(T)-u(P)|}{|T-P|^{\beta}+\frac{2^{\beta}}{5 \sqrt{3}}} \frac{|u(T)-u(Q)|}{|T-Q|^{\beta}} \\
& \leq|u|_{S, \beta}+\frac{2}{5 \sqrt{3}}|u|_{S, \beta}\left(2^{\beta}+1+\frac{3^{\beta / 2}}{2}+2^{(\beta-1)}\right) \\
& \leq 2.2|u|_{S, \beta} \leq 3|u|_{S, \beta} .
\end{aligned}
$$

REMARK 3. We observe that the Hölder seminorm is invariant under translation and rotation. For Lemmas 2-3 and 6-8 we will use this observation and without loss of generality assume that the triangle under consideration $\mathcal{T}$ is located with one vertex at the origin and a second vertex along the $x$-axis. 
REMARK 4 . We observe the following relationship between the vertices that are elements of $V^{n}$ and the vertex $J$ (here $P_{i} \in V^{n}$ ):

$$
u_{n}^{*}(J)=\sum_{i=1}^{M} u\left(P_{i}\right)
$$

and for $2 \leq M \leq 5$ the following estimate holds

$$
\begin{aligned}
\frac{\left|u_{n}^{*}(J)-u\left(P_{i}\right)\right|}{\left|J-P_{i}\right|^{\beta}} \leq \frac{1}{M} \sum_{k=1}^{M} \frac{\left|u\left(P_{k}\right)-u\left(P_{i}\right)\right|}{\left|J-P_{i}\right|^{\beta}} & \leq \frac{1}{M} \sum_{k=1}^{M} \frac{2^{\beta}\left|u\left(P_{k}\right)-u\left(P_{i}\right)\right|}{\left|P_{k}-P_{i}\right|^{\beta}} \\
& \leq \frac{2(M-1)}{M}|u|_{S, \beta} \leq \frac{8}{5}|u|_{S, \beta} .
\end{aligned}
$$

Lemma 2. Given a triangle $\mathcal{T}$ with vertices $P, Q$, and $R$, and a function $u$ defined at the vertices of the prefractal $V^{n}$ such that $|u(A)-u(B)| \leq|u|_{S, \beta}|A-B|^{\beta}$ where $A, B \in V^{n}$ and $\beta \leq 1$. Moreover $P, Q, R \in V^{n}$. Then there exists an extension function $u^{*}$ defined on $\mathcal{T}$ such that

$$
\sup _{X, Y \in \mathcal{T}} \frac{\left|u^{*}(X)-u^{*}(Y)\right|}{|X-Y|^{\beta}} \leq 3|u|_{S, \beta} .
$$

Proof. We begin by letting $u^{*}$ be the affine function defined by the value of $u$ at the three vertices of the triangle. We now check that $u^{*}$ satisfies the estimate (2).

By Remark 3 we may assume $\mathcal{T}$ is a triangle with $P$ located at the origin and $J$ is located on the $x$-axis. It can easily be checked that

$$
\hat{u}^{*}(\hat{X})=\frac{u(Q)-u(P)}{|P-Q|} \hat{x}+\frac{2 u(R)-u(P)-u(Q)}{\sqrt{3}|P-Q|} \hat{y}+u(P) .
$$

Let $X_{1}=\left(x_{1}, y_{1}\right)$ and $X_{2}=\left(x_{2}, y_{2}\right)$ be arbitrary points in $\mathcal{T}$.

$$
\begin{aligned}
\frac{\left|u^{*}\left(X_{1}\right)-u^{*}\left(X_{2}\right)\right|}{\left|X_{1}-X_{2}\right|^{\beta}} & =\frac{\left|\frac{u(Q)-u(P)}{|P-Q|}\left(x_{1}-x_{2}\right)+\frac{2 u(R)-u(P)-u(Q)}{\sqrt{3}|P-Q|}\left(y_{1}-y_{2}\right)\right|}{\left|X_{1}-X_{2}\right|^{\beta}} \\
& \leq \frac{|u(Q)-u(P)|}{|P-Q|^{\beta}}+\frac{|2 u(R)-u(P)-u(Q)|}{\sqrt{3}|P-Q|^{\beta}} \\
& \leq|u|_{S, \beta}+\frac{1}{\sqrt{3}} \frac{|u(R)-u(P)|+|u(R)-u(Q)|}{|P-Q|^{\beta}} \\
& \leq 3|u|_{S, \beta} .
\end{aligned}
$$

Hence $\frac{\left|u^{*}(X)-u^{*}(Y)\right|}{|X-Y|^{\beta}} \leq 3|u|_{S, \beta}$.

The following Lemma holds for any of the secondary sidecar triangles.

Lemma 3. Let $\mathcal{T}$ be a triangle with vertices $P, J$ and $K$ and let $u$ be a function defined at $P$ and $J$. Let us suppose that there exists $\beta \leq 1$ such that 


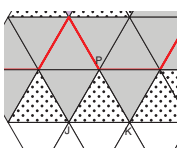

(a)

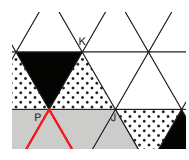

(b)

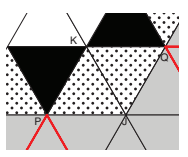

(c)

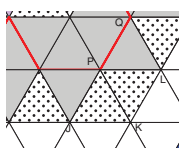

(d)

FIG. 4. Four sample secondary sidecar triangles (Note: the secondary triangles appear dotted, the primary triangles appear gray, and the tertiary triangles appear black).

$\frac{|u(P)-u(J)|}{<} \frac{8}{5}|u|_{S, \beta}|P-J|^{\beta}$ holds. Then there exists a function $u^{*}$ defined for $x \in \mathcal{T}$ such that

$$
\sup _{X, Y \in \mathcal{T}} \frac{\left|u^{*}(X)-u^{*}(Y)\right|}{|X-Y|^{\beta}} \leq 5|u|_{S, \beta}
$$

Proof. First we observe that the secondary sidecar triangles can be classified into four groups as illustrated in Figure 4. In the first scenario (see Figure $4(\mathrm{~A}))$, the value of $u$ at point $K$ is predetermined by a primary sidecar triangle. In the second scenario (see Figure 4(B)), the value of $u^{*}$ at $K$ is set to be $u(K)=\frac{1}{2}(u(P)+u(J))$. In the third scenario (see Figure $4(\mathrm{C})$ ), the value of $u$ at $K$ is set to be $u(K)=\frac{1}{3}(u(P)+u(Q)+u(J))$ where two of these points ( $P$ and $Q$ ) are elements of $V^{n}$ and the third point $J \notin V^{n}$. In the last scenario (see Figure 4(D)), the value of $u$ at $K$ is set to be $u(K)=\frac{1}{3}(u(P)+u(L)+u(J))$ where two of these points $(L$ and $J)$ are sidecar vertices and the third point $P$ is an element of $V^{n}$. In all four scenarios we let $u^{*}$ be the function on $\mathcal{T}$ defined by the values of $u$ at the three vertices. We now check that $u^{*}$ satisfies the estimate (3).

By Remark 3 we may assume $\mathcal{T}$ is a triangle with $P$ located at the origin and $J$ is located on the $x$-axis. It can easily be checked that

$$
u^{*}(X)=\frac{u(J)-u(P)}{|P-J|} x+\frac{2 u(K)-u(P)-u(J)}{\sqrt{3}|P-J|} y+u(P) .
$$

Let $X_{1}=\left(x_{1}, y_{1}\right)$ and $X_{2}=\left(x_{2}, y_{2}\right)$ be arbitrary points in $\mathcal{T}$.

$$
\begin{aligned}
\frac{\left|u^{*}\left(X_{1}\right)-u^{*}\left(X_{2}\right)\right|}{\left|X_{1}-X_{2}\right|^{\beta}} & =\frac{\left|\frac{u(J)-u(P)}{|P-J|}\left(x_{1}-x_{2}\right)+\frac{2 u(K)-u(P)-u(J)}{\sqrt{3}|P-J|}\left(y_{1}-y_{2}\right)\right|}{\left|X_{1}-X_{2}\right|^{\beta}} \\
& \leq \frac{|u(P)-u(J)|}{|P-J|^{\beta}}+\frac{|2 u(K)-u(P)-u(J)|}{\sqrt{3}|P-J|^{\beta}} \\
& \leq \frac{8}{5}|u|_{S, \beta}+\frac{|2 u(K)-u(P)-u(J)|}{\sqrt{3}|P-J|^{\beta}} .
\end{aligned}
$$

We now consider the term $\frac{|2 u(K)-u(P)-u(J)|}{\sqrt{3}|P-J|^{\beta}}$ for each of the scenarios separately. 
CASE 1: $u(K)$ was set by a primary sidecar triangle.

$$
\begin{aligned}
\frac{|2 u(K)-u(P)-u(J)|}{\sqrt{3}|P-J|^{\beta}} & =\frac{|2 u(K)-2 u(P)+u(P)-u(J)|}{\sqrt{3}|P-J|^{\beta}} \\
& \leq \frac{2|u(K)-u(P)|}{\sqrt{3}|K-P|^{\beta}}+\frac{|u(P)-u(J)|}{\sqrt{3}|P-J|^{\beta}} \\
& \leq \frac{1}{\sqrt{3}}\left(\frac{16}{5}+\frac{8}{5}\right)|u|_{S, \beta} \\
& \leq 2.8|u|_{S, \beta} .
\end{aligned}
$$

CASE 2: $u(K)=\frac{1}{2}(u(P)+u(J))$.

$$
\frac{|2 u(K)-u(P)-u(J)|}{\sqrt{3}|P-J|^{\beta}}=\frac{|u(P)+u(J)-u(P)-u(J)|}{\sqrt{3}|P-J|^{\beta}}=0 .
$$

CASE 3: $u(K)=\frac{1}{3}(u(P)+u(J)+u(Q))$.

$$
\begin{aligned}
\frac{|2 u(K)-u(P)-u(J)|}{\sqrt{3}|P-J|^{\beta}} & \leq \frac{1}{3 \sqrt{3}} \frac{|u(Q)-u(P)|+|u(Q)-u(J)|}{|P-J|^{\beta}} \\
& \leq \frac{1}{3 \sqrt{3}}\left(\frac{3^{(\beta / 2)}|u(Q)-u(P)|}{|Q-P|^{\beta}}+\frac{|u(Q)-u(J)|}{|P-J|^{\beta}}\right) \\
& \leq \frac{1}{3 \sqrt{3}}\left(3^{(\beta / 2)}|u|_{S, \beta}+\frac{8}{5}|u|_{S, \beta}\right) \\
& \leq|u|_{S, \beta} .
\end{aligned}
$$

CASE $4: u(K)=\frac{1}{3}(u(P)+u(J)+u(L))$.

$$
\begin{aligned}
\frac{|2 u(K)-u(P)-u(J)|}{\sqrt{3}|P-J|^{\beta}} & \leq \frac{1}{3 \sqrt{3}} \frac{|u(L)-u(P)|+|u(L)-u(J)|}{|P-J|^{\beta}} \\
& \leq \frac{1}{3 \sqrt{3}} \frac{2|u(L)-u(P)|+|u(P)-u(J)|}{|P-J|^{\beta}} \\
& \leq \frac{1}{3 \sqrt{3}}\left(\frac{2|u(L)-u(P)|}{|L-P|^{\beta}}+\frac{|u(P)-u(J)|}{|P-J|^{\beta}}\right) \\
& \leq \frac{1}{3 \sqrt{3}}\left(\frac{16}{5}|u|_{S, \beta}+\frac{8}{5}|u|_{S, \beta}\right) \\
& \leq|u|_{S, \beta} .
\end{aligned}
$$

In all four cases $\frac{|2 u(K)-u(P)-u(J)|}{\sqrt{3}|P-J|^{\beta}} \leq 2.8|u|_{S, \beta}$ and hence $\frac{\left|u^{*}(X)-u^{*}(Y)\right|}{|X-Y|^{\beta}} \leq$ $5|u|_{S, \beta}$. $\mathrm{u}$

The following Lemma holds for any of the tertiary sidecar triangles.

Lemma 4. Let $\mathcal{T}$ be a triangle with vertices $P, J$ and $K$ and let $u$ be a function defined at $P, J$ and $K$. Let us suppose that there exists $\beta \leq 1$ such that

$$
|u(P)-u(J)| \leq 4|u|_{S, \beta}|P-J|^{\beta},
$$


and

$$
|u(P)-u(K)| \leq 4|u|_{S, \beta}|P-K|^{\beta}
$$

hold. Then there exists a function $u^{*}$ defined for $x \in \mathcal{T}$ with $u^{*}(P)=u(P), u^{*}(J)=$ $u(J)$ and $u^{*}(K)=u(K)$ such that

$$
\sup _{X, Y \in \mathcal{T}} \frac{\left|u^{*}(X)-u^{*}(Y)\right|}{|X-Y|^{\beta}} \leq 11|u|_{S, \beta}
$$

REMARK 5. In Equations (4) and (5) the right hand side is less then or equal to $4|u|_{S, \beta}$. This may seem in contradiction to the previous lemma which sets the Hölder constant for a secondary sidecar triangle at $5|u|_{S, \beta}$. The reason for this discrepancy is that those secondary sidecar triangles in the first group (i.e. those triangles where the value of $u_{n}^{*}$ is predetermined by the primary sidecar triangles at all three vertices) never share a side (and hence two vertices) with a tertiary sidecar triangle. This allows us to use the sharper estimates from secondary sidecar triangles of types 2,3 and 4 .

Proof. We begin by letting $u^{*}$ be the affine function defined by the value of $u$ at the three vertices of the triangle. We now check that $u^{*}$ satisfies the estimate (6).

By Remark 3 we may assume $\mathcal{T}$ is a triangle with $P$ located at the origin and $J$ is located on the $x$-axis. It can easily be checked that

$$
u^{*}(X)=\frac{u(J)-u(P)}{|P-J|} x+\frac{2 u(K)-u(P)-u(J)}{\sqrt{3}|P-J|} y+u(P) .
$$

Let $X_{1}=\left(x_{1}, y_{1}\right)$ and $X_{2}=\left(x_{2}, y_{2}\right)$ be arbitrary points in $\mathcal{T}$.

$$
\begin{aligned}
\frac{\left|u^{*}\left(X_{1}\right)-u^{*}\left(X_{2}\right)\right|}{\left|X_{1}-X_{2}\right|^{\beta}} & =\frac{\left|\frac{u(P)-u(J)}{|P-J|}\left(x_{1}-x_{2}\right)+\frac{2 u(K)-u(P)-u(J)}{\sqrt{3}|P-J|}\left(y_{1}-y_{2}\right)\right|}{\left|X_{1}-X_{2}\right|^{\beta}} \\
& \leq 4|u|_{S, \beta}+\frac{1}{\sqrt{3}} \frac{2|u(K)-u(P)|+|u(P)-u(J)|}{|P-J|^{\beta}} \\
& \leq 4|u|_{S, \beta}+\frac{1}{\sqrt{3}} \frac{2|u(K)-u(P)|}{|K-P|^{\beta}}+\frac{1}{\sqrt{3}} \frac{|u(P)-u(J)|}{|P-J|^{\beta}} \\
& \leq 4|u|_{S, \beta}+\frac{3}{\sqrt{3}} 4|u|_{S, \beta} \\
& =|u|_{S, \beta}(4+4 \sqrt{3}) \leq 11|u|_{S, \beta} .
\end{aligned}
$$

Hence $\frac{\left|u^{*}(X)-u^{*}(Y)\right|}{|X-Y|^{\beta}} \leq 11|u|_{S, \beta}$.

The next Lemma considers the case of two triangles each with an affine function defined on them.

Lemma 5. Let $\mathcal{T}_{V}$ and $\mathcal{T}_{W}$ be two triangles such that $\mathcal{T}_{V} \cap \mathcal{T}_{W} \neq \emptyset$. Let $v$ be an affine function defined on $\mathcal{T}_{V}$ and $w$ an affine function defined on $\mathcal{T}_{W}$ 
such that $\left.v\right|_{\mathcal{T}_{V} \cap \mathcal{T}_{W}}=\left.w\right|_{\mathcal{T}_{V} \cap \mathcal{T}_{W}}$. We also require that for some $\beta \leq 1$ there exists constants $C_{V}$ and $C_{W}$ such that $\sup _{X, Y \in \mathcal{T}_{V}}|v(X)-v(Y)| \leq C_{V}|X-Y|^{\beta}$, and $\sup _{X, Y \in \mathcal{T}_{W}}|w(X)-w(Y)| \leq C_{W}|X-Y|^{\beta}$. Define

$$
z(X)= \begin{cases}v(X) & \text { if } X \in \mathcal{T}_{V} \\ w(X) & \text { if } X \in \mathcal{T}_{W} \backslash \mathcal{T}_{V}\end{cases}
$$

and $C_{M}=\max \left(C_{V}, C_{W}\right)$. Then

$$
\sup _{X, Y \in \mathcal{T}_{V} \cup \mathcal{T}_{W}} \frac{|z(X)-z(Y)|}{|X-Y|^{\beta}} \leq 2 C_{M}
$$

Proof. For ease of notation we define $\mathcal{W}:=\mathcal{T}_{V} \cup \mathcal{T}_{W}$. Let $X$ and $Y$ be arbitrary points in $\mathcal{W}$. If $X$ and $Y$ are points in the same triangle the inequality holds trivially. We now consider the case where $X$ and $Y$ are in different triangles and without loss of generality will assume $X \in \mathcal{T}_{V}$ and $Y \in \mathcal{T}_{W}$. We note that if $\mathcal{T}_{V} \cap \mathcal{T}_{W} \neq \emptyset$ then $\mathcal{T}_{V}$ and $\mathcal{T}_{W}$ share either an entire side, or only a single point. In the first case $\overline{X Y} \subset \mathcal{W}$ since every triangle is equilateral and we define $P$ as the intersection of $\overline{X Y}$ and $\mathcal{T}_{V} \cap \mathcal{T}_{W}$. In the second case we define $P=\mathcal{T}_{V} \cap \mathcal{T}_{W}$. We also recall that $v$ and $w$ are affine functions on $\mathcal{T}_{V}$ and $\mathcal{T}_{W}$ respectively. Clearly

$$
\begin{aligned}
\frac{|z(X)-z(Y)|}{|X-Y|^{\beta}} & \leq \frac{|v(X)-v(P)|}{|X-Y|^{\beta}}+\frac{|w(P)-w(Y)|}{|X-Y|^{\beta}} \\
& \leq \frac{C_{V}|X-P|^{\beta}}{|X-Y|^{\beta}}+\frac{C_{W}|P-Y|^{\beta}}{|X-Y|^{\beta}} \\
& =C_{V}\left(\frac{|X-P|}{|X-Y|}\right)^{\beta}+C_{W}\left(\frac{|P-Y|}{|X-Y|}\right)^{\beta} \\
& \leq C_{V}+C_{W} \leq 2 C_{M} .
\end{aligned}
$$

Since $X$ and $Y$ were arbitrary we have shown (7).

Lemma 6. Let $n$ be fixed, $V^{n}$ a prefractal set and given a function $u$ defined on $V^{n}$ such that $|u(P)-u(Q)| \leq|u|_{S, \beta}|P-Q|^{\beta}$ for all $P, Q \in V^{n}$ and for some $\beta \leq 1$. Let $u_{n}^{*}$ be an extension to the domain $\omega$ as defined in Definition 3, then for any $X, Y$ in $T_{S C}^{n}$ the following holds

$$
\frac{\left|u_{n}^{*}(X)-u_{n}^{*}(Y)\right|}{|X-Y|^{\beta}} \leq 66|u|_{S, \beta}
$$

Proof. We define $C_{M S C}$ as the maximum Hölder constant from Lemmas 1-4, therefore $C_{M S C} \leq 11|u|_{S, \beta}$. We also observe that if $X$ and $Y$ are elements of the same triangle $\mathcal{T}$, then (8) holds due to those lemmas. Moreover, if $X$ and $Y$ are elements of two triangles that share either an entire side or a single point, (8) holds by Lemma 5 . We now consider the case where $X$ and $Y$ are elements of $\mathcal{T}_{X}$ and $\mathcal{T}_{Y}$ respectively and that $\mathcal{T}_{X} \cap \mathcal{T}_{Y}=\emptyset$.

We let $P_{X}$ be an element of $V^{n}$ closest to $X$, that is to say $\left|P_{X}-X\right| \leq|R-X|$ for all $R \in V^{n}$ (we know that at least one such element exists). Similarly define $P_{Y}$ 
to be the element of $V^{n}$ closest to $Y$. Let $L$ be the length of the side of a triangle in our mesh (i.e. $L=3^{-n}$ ), therefore we have $\left|P_{X}-X\right| \leq L,\left|P_{Y}-Y\right| \leq L$, $\left|P_{X}-P_{Y}\right| \leq\left|P_{X}-X\right|+|X-Y|+\left|Y-P_{Y}\right|$ and $|X-Y| \geq \frac{\sqrt{3}}{2} L$. We then have,

$$
\begin{aligned}
\left|u_{n}^{*}(X)-u_{n}^{*}(Y)\right| \leq & \left|u_{n}^{*}(X)-u_{n}^{*}\left(P_{X}\right)\right|+\left|u_{n}^{*}\left(P_{X}\right)-u_{n}^{*}\left(P_{Y}\right)\right|+\left|u_{n}^{*}\left(P_{Y}\right)-u_{n}^{*}(Y)\right| \\
& \leq C_{\mathcal{T}_{X}}\left|X-P_{X}\right|^{\beta}+|u|_{S, \beta}\left|P_{X}-P_{Y}\right|^{\beta}+C_{\mathcal{T}_{Y}}\left|P_{Y}-Y\right|^{\beta} \\
& \leq C_{M S C}\left(\left|X-P_{X}\right|^{\beta}+\left|P_{X}-P_{Y}\right|^{\beta}+\left|P_{Y}-Y\right|^{\beta}\right) \\
& \leq C_{M S C}\left[\left(\frac{2}{\sqrt{3}}|X-Y|\right)^{\beta}+\left(\frac{2}{\sqrt{3}}+1+\frac{2}{\sqrt{3}}\right)^{\beta}|X-Y|^{\beta}+\right. \\
& \left.\quad+\left(\frac{2}{\sqrt{3}}|X-Y|\right)^{\beta}\right] \\
& \leq 6 C_{M S C}|X-Y|^{\beta} \\
& \leq 66|u|_{S, \beta}|X-Y|^{\beta} .
\end{aligned}
$$

5. Iterative process. The purpose of this section is to provide a few results necessary to show that $u_{n}^{*}$ satisfies the Hölder estimate when $n \geq 1$. The first three lemmas consider the Hölder estimate of $\mathcal{T} \in T^{n}$ where $n \geq 1$. The fourth lemma is similar to Lemma 6 and shows an estimate for two points in $T_{S C}^{n} \cup T_{T R}^{n}$. The fifth lemma gives a Hölder estimate on each triangle $\mathcal{T}$ of the triangulation $T^{n}$. Finally we consider the Hölder estimate for the function $u_{1}^{*}$ in $\omega$.

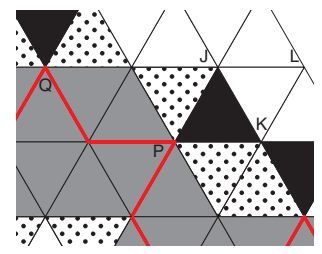

(a)

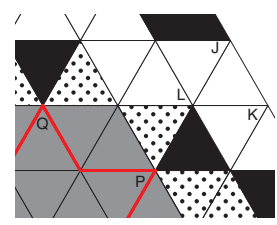

(b)

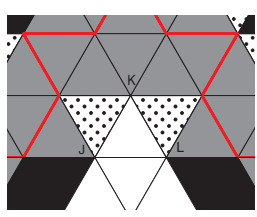

(c)

FIG. 5. The three types of transition triangles considered in Lemmas 7-9. (Recall the transition triangles are white.)

This lemma corresponds to those transition triangles where the value for $u_{n}^{*}$ is determined by the sidecar triangles at two vertices, and the value at the third vertex is determined by the function $u_{n-1}^{*}$. This is shown in Figure 5(A).

Lemma 7. Given a triangle $\mathcal{T}$ with vertices $J, K$, and $L$, a function $u$ defined at points $J, K$ and $L$ such that $|u(J)-u(K)| \leq 11|u|_{S, \beta}|J-K|^{\beta}$, where $\beta \leq 1$. Suppose we are also given two points $P$ and $Q$ in $V^{n}$ such that

$$
\begin{gathered}
|u(P)-u(J)| \leq 11|u|_{S, \beta}|P-J|^{\beta}, \\
|u(P)-u(K)| \leq 11|u|_{S, \beta}|P-K|^{\beta},
\end{gathered}
$$




$$
|u(P)-u(Q)| \leq|u|_{S, \beta}|P-Q|^{\beta},
$$

and

$$
|u(L)-u(Q)| \leq 11|u|_{S, \beta}|L-Q|^{\beta} .
$$

Moreover the following relationships hold: $|J-K|=|P-J|=|P-K|, \quad|J-K| \geq$ $\frac{1}{3}|L-Q|, \quad|J-K| \geq \frac{1}{\sqrt{3}}|P-Q|$. (Again, see Figure 5(A) for a visual representation.) Then, there exists an extension function $u^{*}$ defined on $\mathcal{T}$ such that

$$
\sup _{X, Y \in \mathcal{T}} \frac{\left|u^{*}(X)-u^{*}(Y)\right|}{|X-Y|^{\beta}} \leq 65|u|_{S, \beta} .
$$

REMARK 6. In this lemma the value of $u$ at points $J$ and $K$ is determined by the extension function $u_{n}^{*}$ in the sidecar region and we therefore have the estimate $|u(J)-u(K)| /|J-K|^{\beta} \leq 11|u|_{S, \beta}$. The value of $u$ at point $L$ is determined by the previous extension function $u_{n-1}^{*}$. We use this information to determine the relationship between $L$ and a point in $V^{n-1}$ (i.e. $|u(L)-u(Q)| /|L-Q|^{\beta} \leq 11|u|_{S, \beta}$ ). These relationships drive both the estimates and geometrical assumptions (i.e. the distance between points) in the theorem statement.

REMARK 7. For some transition triangles of this type, the scenario will occur that $P \in V^{n-1}$. In this case there is no need for a distinct point $Q$, so we will set $Q=P$. We observe that all of the estimates and geometrical assumptions are still true in this case.

Proof. We begin by letting $u^{*}$ be the affine function defined by the value of $u$ at the three vertices of the triangle. We now check that $u^{*}$ satisfies $(9)$.

By Remark 3 we may assume $\mathcal{T}$ is a triangle with $P$ located at the origin and $J$ located on the $x$-axis. It can easily be checked that

$$
u^{*}(X)=\frac{u(K)-u(J)}{|K-J|} x+\frac{2 u(L)-u(K)-u(J)}{\sqrt{3}|K-J|} y+u(J) .
$$

Let $X_{1}=\left(x_{1}, y_{1}\right)$ and $X_{2}=\left(x_{2}, y_{2}\right)$ be arbitrary points in $\mathcal{T}$.

$$
\begin{aligned}
& \frac{\left|u^{*}\left(X_{1}\right)-u^{*}\left(X_{2}\right)\right|}{\left|X_{1}-X_{2}\right|^{\beta}} \\
& =\frac{\left|\frac{u(K)-u(J)}{|K-J|}\left(x_{1}-x_{2}\right)+\frac{2 u(L)-u(K)-u(J)}{\sqrt{3}|K-J|}\left(y_{1}-y_{2}\right)\right|}{\left|X_{1}-X_{2}\right|^{\beta}} \\
& \leq 11|u|_{S, \beta}+\frac{2|u(L)-u(Q)|+2|u(Q)-u(P)|+|u(P)-u(J)|+|u(P)-u(K)|}{\sqrt{3}|K-J|^{\beta}} \\
& \leq 11|u|_{S, \beta}+\frac{1}{\sqrt{3}}\left(\frac{2 \cdot 3^{\beta}|u(L)-u(Q)|}{|L-Q|^{\beta}}+\frac{2 \cdot 3^{\beta / 2}|u(Q)-u(P)|}{|Q-P|^{\beta}}\right. \\
& \left.\quad+\frac{|u(P)-u(J)|}{|P-J|^{\beta}}+\frac{|u(P)-u(K)|}{|P-K|^{\beta}}\right) \\
& \leq 11|u|_{S, \beta}+\frac{1}{\sqrt{3}}\left(66|u|_{S, \beta}+4|u|_{S, \beta}+11|u|_{S, \beta}+11|u|_{S, \beta}\right) \\
& \leq 65|u|_{S, \beta} .
\end{aligned}
$$


If $Q=P$ then:

$$
\begin{aligned}
& \frac{\left|u^{*}\left(X_{1}\right)-u^{*}\left(X_{2}\right)\right|}{\left|X_{1}-X_{2}\right|^{\beta}} \\
& \leq \frac{|u(J)-u(K)|}{|K-J|^{\beta}}+\frac{|2 u(L)-u(J)-u(K)|}{\sqrt{3}|K-J|^{\beta}} \\
& \leq 11|u|_{S, \beta}+\frac{2|u(L)-u(P)|+|u(P)-u(J)|+|u(P)-u(K)|}{\sqrt{3}|K-J|^{\beta}} \\
& \leq 11|u|_{S, \beta}+\frac{1}{\sqrt{3}}\left(\frac{2 \cdot 3^{\beta}|u(L)-u(P)|}{|L-P|^{\beta}}+\frac{|u(P)-u(J)|}{|P-J|^{\beta}}+\frac{|u(P)-u(K)|}{|P-K|^{\beta}}\right) \\
& \leq 11|u|_{S, \beta}+\frac{1}{\sqrt{3}}\left(66|u|_{S, \beta}+11|u|_{S, \beta}+11|u|_{S, \beta}\right) \\
& \leq 62|u|_{S, \beta} .
\end{aligned}
$$

This lemma corresponds to those transition triangles where the value of $u_{n}^{*}$ at two vertices is determined by the function $u_{n-1}^{*}$, and the value at the third vertex is determined by the sidecar triangles. See Figure 5(B).

LEMMA 8. Given a triangle $\mathcal{T}$ with vertices $J, K$, and $L$, and a function $u$ defined at points $J, K$ and $L$ such that

$$
\frac{|u(J)-u(K)|}{|J-K|^{\beta}} \leq 11|u|_{S, \beta}
$$

where $\beta \leq 1$. Suppose we are also given two points $P$ and $Q$, such that

$$
\begin{gathered}
|u(P)-u(J)| \leq 11|u|_{S, \beta}|P-J|^{\beta} \\
|u(P)-u(K)| \leq 11|u|_{S, \beta}|P-K|^{\beta} \\
|u(P)-u(Q)| \leq|u|_{S, \beta}|P-Q|^{\beta}
\end{gathered}
$$

and

$$
|u(L)-u(Q)| \leq 11|u|_{S, \beta}|L-Q|^{\beta} .
$$

Moreover the following relationships hold $|J-K|=|J-L|=|L-K|=|L-Q|, \quad \mid J-$ $K\left|\geq \frac{1}{\sqrt{3}}\right| P-Q|, \quad| J-K\left|\geq \frac{1}{3}\right| P-K|, \quad| J-K\left|\geq \frac{1}{3}\right| P-J \mid$. Then there exists an extension function $u^{*}$ defined on $\mathcal{T}$ such that

$$
\sup _{X, Y \in \mathcal{T}} \frac{\left|u^{*}(X)-u^{*}(Y)\right|}{|X-Y|^{\beta}} \leq 65|u|_{S, \beta} .
$$

REMARK 8. In this Lemma the value of $u$ at $L$ is determined by the extension function $u_{n}^{*}$ in the sidecar region. This knowledge drives the assumption $\mid u(L)-$ $u(Q)|/| Q-\left.L\right|^{\beta} \leq 11|u|_{S, \beta}$. The value of $u$ at points $J$ and $K$ are determined by the previous extension function $u_{n-1}^{*}$ and $J$ and $K$ are both elements of the same triangle 
$\mathcal{T} \in T_{S C}^{n-1}$. These relationships drive both the estimates and geometrical assumptions (e.g. the distance between points) in the theorem statement.

REMARK 9. For some transition triangles of this type, the scenario of Lemma 7 may also occur where $P \in V^{n-1}$. As in the previous lemma, we set $Q=P$ and observe that the estimates and geometrical constraints still hold.

Proof. The proof of this lemma proceeds in a manner similar to the proof of Lemma 7.

This lemma corresponds to those transition triangles where the value for $u_{n}^{*}$ is determined by the sidecar triangles at all three vertices. This is shown in Figure 5 (C).

Lemma 9. Given a triangle $\mathcal{T}$ with vertices $J, K$, and $L$, a function $u$ defined at points $J, K$ and $L$ such that

$$
|u(J)-u(K)| \leq 11|u|_{S, \beta}|J-K|^{\beta}
$$

and

$$
|u(K)-u(L)| \leq 11|u|_{S, \beta}|K-L|^{\beta}
$$

where $\beta \leq 1$. See Figure 5(c) for a visual representation. Then, there exists an extension function $u^{*}$ defined on $\mathcal{T}$ such that

$$
\sup _{X, Y \in \mathcal{T}} \frac{\left|u^{*}(X)-u^{*}(Y)\right|}{|X-Y|^{\beta}} \leq 31|u|_{S, \beta} .
$$

Proof. We begin by letting $u^{*}$ be the affine function defined by the value of $u$ at the three vertices of the triangle. We now check that $u^{*}$ satisfies (11).

By Remark 3 we may assume $\mathcal{T}$ is a triangle with $K$ located at the origin and $J$ located on the $x$-axis. It can easily be checked that

$$
u^{*}(X)=\frac{u(K)-u(J)}{|K-J|} x+\frac{2 u(L)-u(K)-u(J)}{\sqrt{3}|K-J|} y+u(J) .
$$

Let $X_{1}=\left(x_{1}, y_{1}\right)$ and $X_{2}=\left(x_{2}, y_{2}\right)$ be arbitrary points in $\mathcal{T}$.

$$
\begin{aligned}
\frac{\left|u^{*}\left(X_{1}\right)-u^{*}\left(X_{2}\right)\right|}{\left|X_{1}-X_{2}\right|^{\beta}} & =\frac{\left|\frac{u(K)-u(J)}{|K-J|}\left(x_{1}-x_{2}\right)+\frac{2 u(L)-u(K)-u(J)}{\sqrt{3}|K-J|}\left(y_{1}-y_{2}\right)\right|}{\left|X_{1}-X_{2}\right|^{\beta}} \\
& \leq 11|u|_{S, \beta}+\frac{2|u(L)-u(K)|+|u(K)-u(J)|}{\sqrt{3}|K-J|^{\beta}} \\
& \leq 11|u|_{S, \beta}+\frac{1}{\sqrt{3}}\left(\frac{2|u(L)-u(K)|}{|L-K|^{\beta}}+\frac{|u(K)-u(J)|}{|K-J|^{\beta}}\right) \\
& \leq 11|u|_{S, \beta}+\frac{1}{\sqrt{3}}\left(22|u|_{S, \beta}+11|u|_{S, \beta}\right) \\
& \leq 31|u|_{S, \beta} .
\end{aligned}
$$

Hence $\frac{\left|u^{*}(X)-u^{*}(Y)\right|}{|X-Y|^{\beta}} \leq 31|u|_{S, \beta}$. 
We now present a lemma similar to Lemma 6 except here we consider two points in $T_{S C}^{n} \cup T_{T R}^{n}$. (The white and light gray region in Figure 1.)

Lemma 10. Let $n$ be fixed, $V^{n}$ a prefractal set and given a function $u$ defined on the set $V^{n}$ such that $|u(P)-u(Q)| \leq|u|_{S, \beta}|P-Q|^{\beta}$ for all $P, Q \in V^{n}$ and for some $\beta \leq 1$. Let $u_{n}^{*}$ be an extension to the domain $\omega$ as defined in Definition 3. Then for any $X, Y$ in $T_{S C}^{n} \cup T_{T R}^{n}$ the following holds

$$
\frac{\left|u_{n}^{*}(X)-u_{n}^{*}(Y)\right|}{|X-Y|^{\beta}} \leq 396|u|_{S, \beta} .
$$

Proof. We begin by defining $C_{M T R}$ as the maximum Hölder constant for triangles in the transition region, specifically $C_{M T R}=65|u|_{S, \beta}$. By $\mathcal{T}_{X}$ and $\mathcal{T}_{Y}$ we refer to the triangles containing the points $X$ and $Y$ respectively. We observe that if $X$ and $Y$ are elements of the same triangle (i.e. $\mathcal{T}_{X}=\mathcal{T}_{Y}$ ) the inequality holds due to Lemmas 1-4 and 7-9. Moreover if $X$ and $Y$ are elements of two triangles that share one or more points, the inequality also holds by application of Lemma 5 . We now consider the case where $\mathcal{T}_{X} \cap \mathcal{T}_{Y}=\emptyset$.

We observe that for any two points $J$ and $K$ in the sidecar region the following inequality holds by Lemma 6

$$
\frac{\left|u_{n}^{*}(J)-u_{n}^{*}(K)\right|}{|J-K|^{\beta}} \leq 66|u|_{S, \beta} .
$$

Therefore if $X, Y \in T_{S C}^{n}$ then (12) holds by Lemma 6 .

We now consider the case with $X, Y \in T_{T R}^{n}$. We let $P_{X}$ be the point in $T_{S C}^{n} \cap \mathcal{T}_{X}$ closest to $X$ (we know at least one such point exists). Similarly define $P_{Y}$ to be the point in $T_{S C}^{n} \cap \mathcal{T}_{Y}$ closest to $Y$. Moreover if $L$ is the length of the side of one triangle in our mesh (i.e. $L=3^{-n}$ ) we have $\left|P_{X}-X\right| \leq L,\left|P_{Y}-Y\right| \leq L,\left|P_{X}-P_{Y}\right| \leq$ $\left|P_{X}-X\right|+|X-Y|+\left|Y-P_{Y}\right|$ and $|X-Y| \geq \frac{\sqrt{3}}{2} L$. We then have,

$$
\begin{aligned}
& \left|u_{n}^{*}(X)-u_{n}^{*}(Y)\right| \\
& \leq\left|u_{n}^{*}(X)-u_{n}^{*}\left(P_{X}\right)\right|+\left|u_{n}^{*}\left(P_{X}\right)-u_{n}^{*}\left(P_{Y}\right)\right|+\left|u_{n}^{*}\left(P_{Y}\right)-u_{n}^{*}(Y)\right| \\
& \leq C_{\mathcal{T}_{X}}\left|X-P_{X}\right|^{\beta}+66|u|_{S, \beta}\left|P_{X}-P_{Y}\right|^{\beta}+C_{\mathcal{T}_{Y}}\left|P_{Y}-Y\right|^{\beta} \\
& \leq \max \left\{C_{M T R}, 66|u|_{S, \beta}\right\}\left(\left|X-P_{X}\right|^{\beta}+\left|P_{X}-P_{Y}\right|^{\beta}+\left|P_{Y}-Y\right|^{\beta}\right) \\
& \leq 66|u|_{S, \beta}\left[\left(\frac{2}{\sqrt{3}}|X-Y|\right)^{\beta}+\left(\frac{4}{\sqrt{3}}+1\right)^{\beta}|X-Y|^{\beta}+\left(\frac{2}{\sqrt{3}}|X-Y|\right)^{\beta}\right] \\
& \leq 396|u|_{S, \beta}|X-Y|^{\beta} .
\end{aligned}
$$

Finally we consider the case with $X \in T_{S C}^{n}$ and $Y \in T_{T R}^{n}$. Again we define $P_{Y}$ to be the point in $T_{S C}^{n} \cap \mathcal{T}_{Y}$ closest to $Y$. (There is no need to define $P_{X}$ since $X \in T_{S C}^{n}$ ). Moreover if $L$ is the length of the side of one triangle in our mesh (i.e. $L=3^{-n}$ ) we have $\left|P_{Y}-Y\right| \leq L,\left|X-P_{Y}\right| \leq|X-Y|+\left|Y-P_{Y}\right|$ and $|X-Y| \geq \frac{\sqrt{3}}{2} L$. We then 
have,

$$
\begin{aligned}
\left|u_{n}^{*}(X)-u_{n}^{*}(Y)\right| & \leq\left|u_{n}^{*}(X)-u_{n}^{*}\left(P_{Y}\right)\right|+\left|u_{n}^{*}\left(P_{Y}\right)-u_{n}^{*}(Y)\right| \\
& \leq 66|u|_{S, \beta}\left|X-P_{Y}\right|^{\beta}+C_{\mathcal{T}_{Y}}\left|P_{Y}-Y\right|^{\beta} \\
& \leq \max \left\{C_{M T R}, 66|u|_{S, \beta}\right\}\left(\left|X-P_{Y}\right|^{\beta}+\left|P_{Y}-Y\right|^{\beta}\right) \\
& \leq 66|u|_{S, \beta}\left[\left(1+\frac{2}{\sqrt{3}}\right)^{\beta}|X-Y|^{\beta}+\left(\frac{2}{\sqrt{3}}|X-Y|\right)^{\beta}\right] \\
& \leq 220|u|_{S, \beta}|X-Y|^{\beta} .
\end{aligned}
$$

Therefore for $X, Y \in T_{S C}^{n} \cup T_{T R}^{n}$ estimate (12) holds. $\square$

This lemma considers the Hölder estimate on an individual triangle $\mathcal{T}$ of the triangulation $T^{n}$ of $\omega$.

Lemma 11. Let $T^{n}$ be the induced triangulation of $\omega$ for the extension function $u_{n}^{*}$, then for any $X, Y$ in a single triangle $\mathcal{T} \in T^{n}$ the following holds

$$
\frac{\left|u_{n}^{*}(X)-u_{n}^{*}(Y)\right|}{|X-Y|^{\beta}} \leq 65|u|_{S, \beta} .
$$

Proof. If $\mathcal{T} \in T_{S C}^{n} \cup T_{T R}^{n}$ the inequality holds by Lemmas 1-4 and 7-9. We now consider the case when $\mathcal{T} \in T_{E X}^{n}$ (the dark region of Figure 1). We identify by $m$ the iteration where the value of $u_{n}^{*}(\mathcal{T})$ was last set, that is to say $u_{m}^{*}(X) \neq u_{m-1}^{*}(X)$ for some $X \in \mathcal{T}$ but $u_{m}^{*}(X)=u_{m+i}^{*}(X)$ where $1 \leq i \leq n-m$ for all $X \in \mathcal{T}$. We identify by $\mathcal{T}_{m}$ the triangle from the triangulation $T^{m}$ which contains $\mathcal{T}$.

By construction of the extension function in $\omega, \mathcal{T}_{m} \in T_{S C}^{m} \cup T_{T R}^{m}$. Therefore for $A, B \in \mathcal{T}_{m}$ we have $\frac{\left|u_{m}^{*}(A)-u_{m}^{*}(B)\right|}{|A-B|^{\beta}} \leq 65|u|_{S, \beta}$. Since $\mathcal{T} \subset \mathcal{T}_{m}$, for $X, Y$ in $\mathcal{T}$ we have $\frac{\left|u_{m}^{*}(X)-u_{m}^{*}(Y)\right|}{|X-Y|^{\beta}} \leq 65|u|_{S, \beta}$. Therefore for $X, Y \in \mathcal{T}$ :

$$
\frac{\left|u_{n}^{*}(X)-u_{n}^{*}(Y)\right|}{|X-Y|^{\beta}}=\frac{\left|u_{m}^{*}(X)-u_{m}^{*}(Y)\right|}{|X-Y|^{\beta}} \leq 65|u|_{S, \beta} .
$$

The next Lemma considers the step $u_{0}^{*}$ to $u_{1}^{*}$ illustrated in Figure 1 .

Lemma 12. Let $n=1$ and given a function $u$ defined on the prefractal set $V^{1}$ such that $|u(P)-u(Q)| \leq|u|_{S, \beta}|P-Q|^{\beta}$ for all $P, Q \in V^{1}$ and for some $\beta \leq 1$. Let $u_{1}^{*}$ be the first extension to the subdomain $\omega$ as defined in Definition 3, then for any $X, Y$ in $\omega$ the following holds

$$
\frac{\left|u_{1}^{*}(X)-u_{1}^{*}(Y)\right|}{|X-Y|^{\beta}} \leq 820|u|_{S, \beta} .
$$

Proof. First we make a few definitions. We define $C_{M S C}$ as the maximum sidecar constant from Lemmas $1-4$, specifically $C_{M S C} \leq 11|u|_{S, \beta}$. We define $C_{M T R}$ as the maximum transition triangle constant from Lemmas 7-9, specifically 
$C_{M T R} \leq 65|u|_{S, \beta}$. Clearly if $X, Y$ are elements of the same triangle, or if $X, Y$ are in two separate triangles that share one or more points, then (15) holds. If $X, Y \in T_{S C}^{n} \cup T_{T R}^{n}$ then (15) holds by Lemma 10. We now consider the case where $X$ is in $T_{S C}^{n} \cup T_{T R}^{n}$ and $Y$ is in $T_{E X}^{n}=\omega \backslash\left(T_{S C}^{n} \cup T_{T R}^{n}\right)$.

We let $P_{X}$ be the point in $T_{S C}^{n} \cap \mathcal{T}_{X}$ such that $\left|P_{X}-X\right|$ is minimized. (Note that if $X \in T_{S C}^{n}$, than $P_{X}=X$.) Moreover we identify by $Q_{X}$ the element of $V^{1}$ closest to the point $P_{X}$ and by $Q_{Y}$ the element of $V^{0}$ closest to $Y$. (We recall that when $u_{0}^{*}$ was constructed every point in $\omega$ was an element of the sidecar region). We let $L$ be the length of the side of a triangle in induced triangulation $T^{1}$. (i.e. $L=|A-B| / 3=1 / 3$ ) and observe that $\left|X-P_{X}\right| \leq L,\left|P_{X}-Q_{X}\right| \leq L$ and $\left|Q_{Y}-Y\right| \leq 3 L$. We also observe that $|X-Y| \geq \frac{\sqrt{3}}{2} L$. We then have:

$$
\begin{aligned}
& \left|u_{1}^{*}(X)-u_{1}^{*}(Y)\right| \\
& =\left|u_{1}^{*}(X)-u_{0}^{*}(Y)\right| \\
& \leq C_{M T R}\left|X-P_{X}\right|^{\beta}+C_{M S C}\left|P_{X}-Q_{X}\right|^{\beta}+|u|_{S, \beta}\left|Q_{X}-Q_{Y}\right|^{\beta}+C_{M S C}\left|Q_{Y}-Y\right|^{\beta} \\
& \leq \max \left\{C_{M T R}, C_{M S C},|u|_{S, \beta}\right\}\left(\left|X-P_{X}\right|^{\beta}+\left|P_{X}-Q_{X}\right|^{\beta}+\left|Q_{X}-Q_{Y}\right|^{\beta}+\left|Q_{Y}-Y\right|^{\beta}\right) \\
& \leq 65|u|_{S, \beta}\left[\left(\frac{2}{\sqrt{3}}|X-Y|\right)^{\beta}+\left(\frac{2}{\sqrt{3}}|X-Y|\right)^{\beta}+\left|Q_{X}-Q_{Y}\right|^{\beta}+\left(\frac{6}{\sqrt{3}}|X-Y|\right)^{\beta}\right] \\
& \leq 65|u|_{S, \beta}\left[2\left(\frac{2}{\sqrt{3}}\right)^{\beta}|X-Y|^{\beta}+\left(2\left(\frac{2}{\sqrt{3}}\right)+1+\left(\frac{6}{\sqrt{3}}\right)\right)^{\beta}|X-Y|^{\beta}+\right. \\
& \left.\quad+\left(\frac{6}{\sqrt{3}}\right)^{\beta}|X-Y|^{\beta}\right] \\
& \leq 65|u|_{S, \beta}\left[4\left(\frac{2}{\sqrt{3}}\right)^{\beta}+1^{\beta}+2\left(\frac{6}{\sqrt{3}}\right)^{\beta}\right]|X-Y|^{\beta} \\
& \leq 820|u|_{S, \beta}|X-Y|^{\beta} .
\end{aligned}
$$

\section{Main results.}

Proposition 2. Given a prefractal Koch set, $V^{n}$, with iteration number $n \geq 1$ and a function $u$ defined at the vertices of the curve that satisfies $|u(X)-u(Y)| \leq$ $|u|_{S, \beta}|X-Y|^{\beta}$ for all $X, Y \in V^{n}$ and for some $\beta \leq 1$. Let $u_{n}^{*}$ be the $n$th extension to the domain $\omega$. Then for any $X, Y$ in $\omega$ the following holds

$$
\frac{\left|u_{n}^{*}(X)-u_{n}^{*}(Y)\right|}{|X-Y|^{\beta}} \leq 1300|u|_{S, \beta} .
$$

REMARK 10. For any $X$ in $T_{E X}^{n}=\omega \backslash\left(T_{S C}^{n} \cup T_{T R}^{n}\right)$ (i.e. the dark region in Figure 1) $u_{n}^{*}(X)=u_{n-1}^{*}(X)$. This observation will be especially relevant in the proof below.

Proof. By Lemma 12, (16) is true for the extension function $u_{1}^{*}$. Let $u_{n-1}^{*}$ be the $(n-1)$ th extension to the domain $\omega$ and we assume that the following estimate holds for all $X, Y$ in $\omega$

$$
\frac{\left|u_{n-1}^{*}(X)-u_{n-1}^{*}(Y)\right|}{|X-Y|^{\beta}} \leq 1300|u|_{S, \beta} .
$$


We now show that the estimate holds for $u_{n}^{*}$ with identical constant on the right hand side.

For ease of notation we set $C_{M S C}$ to be the maximum sidecar constant from Lemmas 1-4, specifically $C_{M S C} \leq 11|u|_{S, \beta}$ and $C_{M T R}$ to be the maximum transition triangle constant from Lemmas $7-9$, specifically $C_{M T R} \leq 65|u|_{S, \beta}$. We also recall the definition of $\omega$ as the polygonal domain with vertices $A=(0,0), B=(1,0)$, $C=\left(\frac{1}{2}, \frac{\sqrt{3}}{2}\right), D=\left(\frac{1}{2},-\frac{\sqrt{3}}{2}\right)$.

By Lemma 10 for $X, Y \in T_{S C}^{n} \cup T_{T R}^{n}$ estimate (16) holds. We now consider the case with $X \in T_{S C}^{n} \cup T_{T R}^{n}$ and $Y \in T_{E X}^{n}$. From Lemma 11, any two points $X^{\prime}, Y^{\prime}$ in a triangle $\mathcal{T}$ of the triangulation $T^{n}$ of $\omega$ the following estimate

$$
\frac{\left|u_{n}^{*}\left(X^{\prime}\right)-u_{n}^{*}\left(Y^{\prime}\right)\right|}{\left|X^{\prime}-Y^{\prime}\right|^{\beta}} \leq 65|u|_{S, \beta}
$$

holds. Combining this estimate with Lemma 5, (16) holds for any two triangles in $T^{n}$ that share one or more points. We now assume that $X$ and $Y$ are in two distinct triangles which do not share any points.

We begin by observing that the values of $u_{n}^{*}$ in $T_{E X}^{n}$ are historically stratified. This stratification can be observed by noting that the values of $u_{n}^{*}$ at points in this region are layered according to when the value of $u_{n}^{*}$ was last set. Specifically $T_{E X}^{n}$ can be broken into subregions where the value of $u_{n}^{*}$ was last set at iterations $n-1, n-2, \ldots, 0$. The stratification for $n=3$ is shown in Figure 6 .

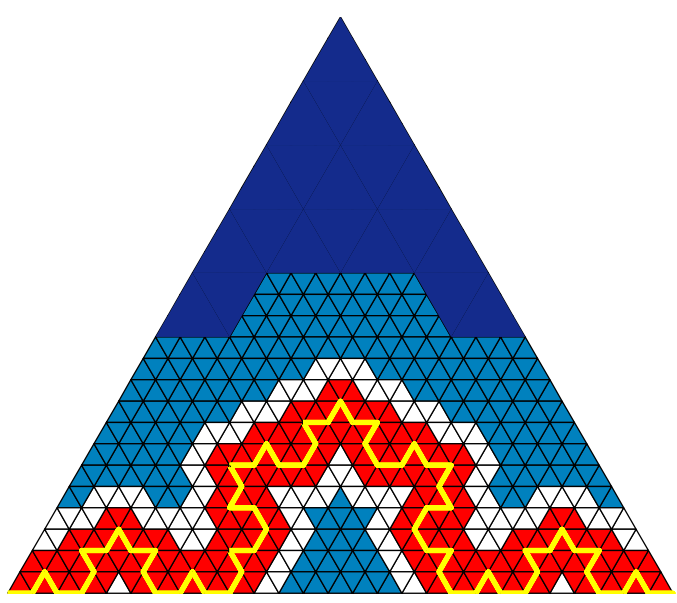

FIG. 6. In this figure the historical stratification is seen.

We identify by $m_{X}=n$ the current iteration (recall $X \in T_{S C}^{n} \cup T_{T R}^{n}$ so the value of $u_{n}^{*}(X)$ is set at this iteration). We identify by $\mathcal{T}_{X}$ the triangle from $T^{m_{X}}$ which contains the point $X$. We let $P_{X}$ be the point in $T_{S C}^{m_{X}} \cap \mathcal{T}_{X}$ such that $\left|P_{X}-X\right|$ 
is minimized. (We note that if $X \in T_{S C}^{m_{X}}$ then $P_{X}=X$ ). We also identify by $Q_{X}$ the point in $V^{m_{X}}$ such that $\left|Q_{X}-P_{X}\right|$ is minimized. We let $L$ be the length of the side in $T^{m_{X}}$ (i.e. $L=3^{-n}$ ) and observe that $\left|X-P_{X}\right| \leq 3^{\left(n-m_{X}\right)} L=L$ and $\left|P_{X}-Q_{X}\right| \leq 3^{\left(n-m_{X}\right)} L=L$. (See Figure 7(a)).

Similarly for $\mathrm{Y}$, we identify by $m_{Y}$ the iteration where the value of $u_{n}^{*}(Y)$ was last set, that is to say $u_{m_{Y}}^{*}(Y) \neq u_{m_{Y}-1}^{*}(Y)$ but $u_{m_{Y}}^{*}(Y)=u_{m_{Y}+i}^{*}(Y)$ where $1 \leq i \leq n-m_{Y}$. We identify by $\mathcal{T}_{Y}$ the triangle from $T^{m_{Y}}$ where the value of $Y$ was last set. We let $P_{Y} \in T_{S C}^{m_{Y}} \cap \mathcal{T}_{Y}$ such that $\left|P_{Y}-Y\right|$ is minimized. (We note that if $Y \in T_{S C}^{m_{Y}}$ then $P_{Y}=Y$ ). We also identify by $Q_{Y}$ the point in $V^{m_{Y}}$ that minimizes $\left|Q_{Y}-P_{Y}\right|$. Using the above definition of $L$ (i.e. $L=3^{-n}$ ) we observe that $\left|Y-P_{Y}\right| \leq 3^{\left(n-m_{Y}\right)} L$ and $\left|P_{Y}-Q_{Y}\right| \leq 3^{\left(n-m_{Y}\right)} L$. See Figure $7(\mathrm{~b})$.

We note that $V^{m_{Y}} \subset V^{m_{X}}=V^{n}$ and now make an important observation concerning the relationship between $|X-Y|$ and $n-m_{Y}$ :

$$
|X-Y| \geq 3^{\left(n-m_{Y}-1\right)} \frac{\sqrt{3}}{2} L .
$$

Having made this observation:

$$
\begin{aligned}
& \left|u_{n}^{*}(X)-u_{n}^{*}(Y)\right| \\
& =\left|u_{m_{X}}^{*}(X)-u_{m_{Y}}^{*}(Y)\right| \\
& \leq\left|u_{m_{X}}^{*}(X)-u_{m_{X}}^{*}\left(P_{X}\right)\right|+\left|u_{m_{X}}^{*}\left(P_{X}\right)-u_{m_{X}}^{*}\left(Q_{X}\right)\right|+\left|u_{m_{X}}^{*}\left(Q_{X}\right)-u_{m_{Y}}^{*}\left(Q_{Y}\right)\right| \\
& +\left|u_{m_{Y}}^{*}\left(Q_{Y}\right)-u_{m_{Y}}^{*}\left(P_{Y}\right)\right|+\left|u_{m_{Y}}^{*}\left(P_{Y}\right)-u_{m_{Y}}^{*}(Y)\right| \\
& \leq \max \left\{C_{M T R}, C_{M S C},|u|_{S, \beta}\right\}\left(\left|X-P_{X}\right|^{\beta}+\left|P_{X}-Q_{X}\right|^{\beta}+\left|Q_{X}-Q_{Y}\right|^{\beta}\right. \\
& \left.+\left|Q_{Y}-P_{Y}\right|^{\beta}+\left|P_{Y}-Y\right|^{\beta}\right) \\
& \leq 65|u|_{S, \beta}\left[\left(\frac{2}{3^{\left(n-m_{Y}-1\right)} \sqrt{3}}|X-Y|\right)^{\beta}+\left(\frac{2}{3^{\left(n-m_{Y}-1\right)} \sqrt{3}}|X-Y|\right)^{\beta}+\left|Q_{X}-Q_{Y}\right|^{\beta}+\right. \\
& \left.+\left(\frac{2 \cdot 3^{\left(n-m_{Y}\right)}}{3^{\left(n-m_{Y}-1\right)} \sqrt{3}}|X-Y|\right)^{\beta}+\left(\frac{2 \cdot 3^{\left(n-m_{Y}\right)}}{3^{\left(n-m_{Y}-1\right)} \sqrt{3}}|X-Y|\right)^{\beta}\right] \\
& \leq 65|u|_{S, \beta}\left[2\left(\frac{2}{\sqrt{3}}|X-Y|\right)^{\beta}+\left|Q_{X}-Q_{Y}\right|^{\beta}+2\left(\frac{6}{\sqrt{3}}|X-Y|\right)^{\beta}\right] \\
& \leq 65|u|_{S, \beta}\left[2\left(\frac{2}{\sqrt{3}}\right)^{\beta}|X-Y|^{\beta}+\left(2\left(\frac{2}{\sqrt{3}}\right)+1+2\left(\frac{6}{\sqrt{3}}\right)\right)^{\beta}|X-Y|^{\beta}\right. \\
& \left.+2\left(\frac{6}{\sqrt{3}}\right)^{\beta}|X-Y|^{\beta}\right] \\
& \leq 65|u|_{S, \beta}\left[4\left(\frac{2}{\sqrt{3}}\right)^{\beta}+1^{\beta}+4\left(\frac{6}{\sqrt{3}}\right)^{\beta}\right]|X-Y|^{\beta} \\
& \leq 65|u|_{S, \beta} \cdot 20|X-Y|^{\beta} \\
& \leq 1300|X-Y|^{\beta} \text {. }
\end{aligned}
$$

One final case remains to be considered, the case with $X, Y \in T_{E X}^{n}$ (the dark region of Figure 1). By Remark 10 we know that for all $X, Y \in T_{E X}^{n}$ that $u_{n}^{*}(X)=$ 
$u_{n-1}^{*}(X)$ and $u_{n}^{*}(Y)=u_{n-1}^{*}(Y)$. We then apply our induction assumption (i.e. that the estimate holds for the $(n-1) t h$ iteration), thereby giving us $\frac{\left|u_{n}^{*}(X)-u_{n}^{*}(Y)\right|}{|X-Y|^{\beta}} \leq$ $1300|u|_{S, \beta}$ for all $X, Y \in T_{E X}^{n}$. Combining the estimates for each specific case we arrive at $\frac{\left|u_{n}^{*}(X)-u_{n}^{*}(Y)\right|}{|X-Y|^{\beta}} \leq 1300|u|_{S, \beta}$ for all $X, Y \in \omega$. $\mathbf{}$

Proposition 3. For every $n$ and every $u_{n} \in C^{\beta}\left(V^{n}\right)$ we construct a linear extension operator $\Pi_{n}$ that brings functions defined on $V^{n}$ to functions defined on $\Omega$ which have the following properties for every $0<\beta \leq 1$ :

1. $u_{n} \in C^{\beta}\left(V^{n}\right) \mapsto u_{n}^{*} \in C^{\beta}(\Omega)$,

2. $\left\|\Pi_{n} u_{n}\right\|_{\Omega, \beta} \leq 1302\|u\|_{S, \beta}$.

Proof. By Proposition 2 we know that for $X, Y$ in $\omega$ and $u_{n}^{*}$ the following inequality holds:

$$
\frac{\left|u_{n}^{*}(X)-u_{n}^{*}(Y)\right|}{|X-Y|^{\beta}} \leq 1300|u|_{S, \beta} .
$$

First we recall the definition of the domain $\gamma$ found in Section 3.0.2 as a convex polygonal domain which contains $\omega$. Section 3.0.2 also details the methodology to extend the function $u_{n}^{*}$ to $\gamma$ and then to $\Omega$. Let $X_{1}$ and $X_{2}$ be arbitrary points in $\gamma$. Then

$$
u_{n}^{*}\left(X_{1}\right)=v_{n}^{*}\left(X_{1}\right) \eta\left(X_{1}\right)=v_{n}^{*}\left(X_{1}^{\prime}\right) \eta\left(X_{1}\right)
$$

where $X_{1}^{\prime} \in \omega$. Similarly,

$$
u_{n}^{*}\left(X_{2}\right)=v_{n}^{*}\left(X_{2}\right) \eta\left(X_{2}\right)=v_{n}^{*}\left(X_{2}^{\prime}\right) \eta\left(X_{2}\right)
$$

where $X_{2}^{\prime} \in \omega$. Moreover $\left|X_{1}-X_{2}\right| \geq\left|X_{1}^{\prime}-X_{2}^{\prime}\right|$ and by geometric arguments $\frac{\left|\eta\left(X_{1}\right)-\eta\left(X_{2}\right)\right|}{\left|X_{1}^{\prime}-X_{2}^{\prime}\right|} \leq \frac{2}{\sqrt{3}}$. Therefore,

$$
\begin{aligned}
\frac{\left|u_{n}^{*}\left(X_{1}\right)-u_{n}^{*}\left(X_{2}\right)\right|}{\left|X_{1}-X_{2}\right|^{\beta}} & =\frac{\left|v_{n}^{*}\left(X_{1}^{\prime}\right) \eta\left(X_{1}\right)-v_{n}^{*}\left(X_{2}^{\prime}\right) \eta\left(X_{2}\right)\right|}{\left|X_{1}-X_{2}\right|^{\beta}} \\
& \leq \frac{\left|v_{n}^{*}\left(X_{1}^{\prime}\right) \eta\left(X_{1}\right)-v_{n}^{*}\left(X_{2}^{\prime}\right) \eta\left(X_{2}\right)\right|}{\left|X_{1}^{\prime}-X_{2}^{\prime}\right|^{\beta}} \\
& \leq \frac{\eta\left(X_{1}\right)\left|v_{n}^{*}\left(X_{1}^{\prime}\right)-v_{n}^{*}\left(X_{2}^{\prime}\right)\right|}{\left|X_{1}^{\prime}-X_{2}^{\prime}\right|^{\beta}}+\frac{v^{*} n\left(X_{2}^{\prime}\right)\left|\eta\left(X_{1}^{\prime}\right)-\eta\left(X_{2}^{\prime}\right)\right|}{\left|X_{1}^{\prime}-X_{2}^{\prime}\right|^{\beta}} \\
& \leq \frac{\left|v_{n}^{*}\left(X_{1}^{\prime}\right)-v_{n}^{*}\left(X_{2}^{\prime}\right)\right|}{\left|X_{1}^{\prime}-X_{2}^{\prime}\right|^{\beta}}+\sup _{X \in S}|u(X)| \frac{\left|\eta\left(X_{1}^{\prime}\right)-\eta\left(X_{2}^{\prime}\right)\right|}{\left|X_{1}^{\prime}-X_{2}^{\prime}\right|^{\beta}} \\
& \leq 1300|u|_{S, \beta}+\sup _{X \in S}|u(X)| \frac{\left|\eta\left(X_{1}^{\prime}\right)-\eta\left(X_{2}^{\prime}\right)\right|}{\left|X_{1}^{\prime}-X_{2}^{\prime}\right|}\left|X_{1}^{\prime}-X_{2}^{\prime}\right|^{\beta} \\
& \leq 1300|u|_{S, \beta}+\sup _{X \in S}|u(X)| \frac{2}{\sqrt{3}}(\sqrt{3})^{\beta} \\
& \leq 1300|u|_{S, \beta}+2\|u\|_{S, \beta} \\
& \leq 1302\|u\|_{S, \beta} .
\end{aligned}
$$




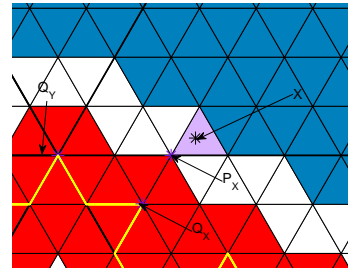

(a) Identification for $X$

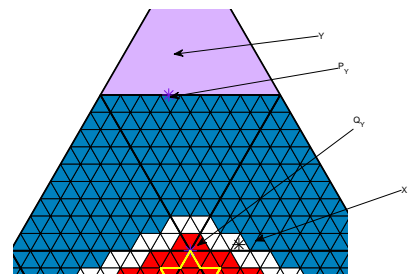

(b) Identification for $Y$

FIG. 7. The identification of triangles $\mathcal{T}_{X}$ and $\mathcal{T}_{Y}$ (the light gray triangles) and points $P_{X}, P_{Y}$, $Q_{X}$ and $Q_{Y}$.

Since $u_{n}^{*}$ is identically zero in $\mathbb{R}^{2} \backslash \gamma$ we have an extension function $u_{n}^{*}$ that is Hölder continuous and satisfies the estimate

$$
\left|u_{n}^{*}\right|_{\Omega, \beta} \leq 1302\|u\|_{S, \beta}
$$

Therefore $\frac{\left|u_{n}^{*}(X)-u_{n}^{*}(Y)\right|}{|X-Y|^{\beta}} \leq 1300|u|_{S, \beta}$ for all $X \in T_{S C}^{n} \cup T_{T R}^{n}$, and $Y \in T_{T R}^{n}$.

We now present the proof of the Theorem 1 .

Proof. We claim $\Pi: C^{\beta}(S) \mapsto C^{\beta}(\Omega)$. We begin by showing that $\|\Pi u\|_{\Omega, \beta} \leq$ $C_{1}\|u\|_{S, \beta}$ and $\Pi u=\lim _{n \rightarrow \infty} \Pi_{n}\left(\left.u\right|_{V^{n}}\right)$ uniformly in $\Omega$. We let $u$ be a Hölder continuous function defined on the fractal curve $S$ with exponent $\beta \leq 1$ and constant $C_{0}=|u|_{S, \beta}$, (i.e. $|u(x)-u(y)| \leq C_{0}|x-y|^{\beta} \quad \forall x, y \in S$ ). We recall that for a given $n, V^{n}$ is a subset of the fractal $S$, and thus given a Hölder continuous function $u$ on $S$ we have $u_{n} \in C^{\beta}\left(V^{n}\right)$. From Proposition 3 we can construct an extension function $u_{n}^{*}$ on $\Omega$ such that

$$
\frac{\left|u_{n}^{*}(X)-u_{n}^{*}(Y)\right|}{|X-Y|^{\beta}} \leq 1302\|u\|_{S, \beta}
$$

for all $X, Y \in \Omega$. (Here we emphasize that the estimate is independent of $n$.) Therefore each extension $u_{n}^{*}$ is equicontinuous on $\Omega$. Moreover we know $u$ is bounded on $S$ with $|u(X)| \leq\|u\|_{S, \beta}$ for all $X \in S$. We therefore can say that $\left|u_{n}^{*}(X)\right| \leq\|u\|_{S, \beta}$ for all $X \in \Omega$. By the Arzelà-Ascoli theorem there exists a subsequence which converges uniformly to a Hölder continuous function $u^{*}$. We now easily show that uniform convergence preserves the Hölder exponent $\beta$ and constant $1302\|u\|_{S, \beta}$.

$$
\frac{\left|u^{*}(X)-u^{*}(Y)\right|}{|X-Y|^{\beta}} \leq \frac{\left|u^{*}(X)-u_{n}^{*}(X)\right|+\left|u_{n}^{*}(X)-u_{n}^{*}(Y)\right|+\left|u_{n}^{*}(Y)-u^{*}(Y)\right|}{|X-Y|^{\beta}}
$$

Since $u_{n}^{*}$ converges to $u$ uniformly given $\epsilon>0$ there exists $N$ such that for all $n \geq$ $N, \quad\left|u^{*}(X)-u_{n}^{*}(X)\right|<\epsilon$ for all $X$ in $\Omega$. Therefore (20) is less then or equal to

$$
\frac{2 \epsilon+\left|u_{n}^{*}(X)-u_{n}^{*}(Y)\right|}{|X-Y|^{\beta}} .
$$


Since $\epsilon$ can be arbitrarily small we have

$$
\frac{\left|u^{*}(X)-u^{*}(Y)\right|}{|X-Y|^{\beta}} \leq 1302\|u\|_{S, \beta} .
$$

Hence we have a function $u^{*}$ which is Hölder continuous everywhere in $\Omega$ and satisfies the estimate

$$
\left|u^{*}\right|_{\bar{\Omega}, \beta} \leq 1302\|u\|_{S, \beta} .
$$

Moreover, $u^{*}(X)=u(X)$ for all $X \in V^{\infty}$ and, due to the density of $V^{\infty}$ in $S$, corresponds with $u$ at every point on $S$.

We now show $\sup _{X \in \Omega}\left|\Pi_{n} u_{n}(X)-\Pi_{n+p} u_{n+p}(X)\right| \leq C_{2}\|u\|_{S, \beta} 3^{-n}$. Fix $p>0$. We observe that by construction, two consecutive extension functions $\left(u_{n}^{*}\right.$ and $\left.u_{n+1}^{*}\right)$ differ on $\omega$ only in the sidecar and transition triangle regions of the $u_{n+1}^{*}$ extension. We will assume that $X \in T_{S C}^{n} \cup T_{T R}^{n}$ or in the equivalent area of $\gamma$. Moreover by $Y$ we denote the element of $V^{n}$ closest to $X$.

$$
\begin{aligned}
\left|u_{n}^{*}(X)-u_{n+p}^{*}(X)\right| & \leq\left|u_{n}^{*}(X)-u(Y)\right|+\left|u(Y)-u_{n+p}^{*}(X)\right| \\
& \leq 2620\|u\|_{S, \beta}|X-Y|^{\beta} \\
& \leq 2620\|u\|_{S, \beta} 3^{-\beta n} .
\end{aligned}
$$

\section{REFERENCES}

[1] Y. Brudnyi and P. Shvartsman, Generalizations of Whitney's extension theorem, Internat. Math. Res. Notices, (3):129 ff., approx. 11 pp. (electronic), 1994.

[2] Y. BRudnyi And P. ShVARTSMan, The Whitney problem of existence of a linear extension operator, J. Geom. Anal., 7:4 (1997), pp. 515-574.

[3] C. Fefferman, $C^{m}$ extension by linear operators, Ann. of Math. (2), 166:3 (2007), pp. $779-835$.

[4] C. Fefferman, Extension of $C^{m, \omega}$-smooth functions by linear operators, Rev. Mat. Iberoam., 25:1 (2009), pp. 1-48.

[5] C. Fefferman, Whitney's extension problems and interpolation of data, Bull. Amer. Math. Soc. (N.S.), 46:2 (2009), pp. 207-220.

[6] C. Fefferman And B. Klartag, An example related to Whitney extension with almost minimal $C^{m}$ norm, Rev. Mat. Iberoam., 25:2 (2009), pp. 423-446.

[7] A. Jonsson, Besov spaces on closed subsets of $\mathbf{R}^{n}$, Trans. Amer. Math. Soc., 341:1 (1994), pp. $355-370$.

[8] A. Jonsson And H. Wallin, Function spaces on subsets of $\mathbf{R}^{n}$, Math. Rep., 2:1 (1984), : $x i v+221$.

[9] A. Jonsson and H. Wallin, The dual of Besov spaces on fractals, Studia Math., 112:3 (1995), pp. 285-300.

[10] M. R. LANCIA AND M. A. Vivaldi, Asymptotic convergence of transmission energy forms, Adv. Math. Sci. Appl., 13:1 (2003), pp. 315-341.

[11] U. Mosco And M. A. VIVALdi, An example of fractal singular homogenization, Georgian Math. J., 14:1 (2007), pp. 169-193.

[12] E. VACCA, Galerkin Approximation for Highly Conductive Layers, PhD thesis, Universita degli Studi di Roma "LaSapienza", 2005.

[13] H. Whitney, Analytic extensions of differentiable functions defined in closed sets, Trans. Amer. Math. Soc., 36:1 (1934), pp. 63-89.

[14] H. Whitney, Differentiable functions defined in closed sets, I, Trans. Amer. Math. Soc., 36:2 (1934), pp. 369-387.

[15] H. Whitney, Functions differentiable on the boundaries of regions, Ann. of Math. (2), 35:3 (1934), pp. 482-485. 